\title{
Timing regulation in a network reduced from voltage-gated equations to a one-dimensional map ${ }^{\star}$
}

\author{
Thomas LoFaro, Nancy Kopell \\ ${ }^{1}$ Department of Pure and Applied Mathematics, Washington State University, \\ Pullman, WA 99163-3113, USA. e-mail: lofaro@trout.math.wsu.edu \\ ${ }^{2}$ Department of Mathematics, Boston University, Boston, MA 02215, USA. \\ e-mail: nk@math.bu.edu
}

Received: 19 May 1997/Revised version: 6 April 1998

\begin{abstract}
We discuss a method by which the dynamics of a network of neurons, coupled by mutual inhibition, can be reduced to a onedimensional map. This network consists of a pair of neurons, one of which is an endogenous burster, and the other excitable but not bursting in the absence of phasic input. The latter cell has more than one slow process. The reduction uses the standard separation of slow/fast processes; it also uses information about how the dynamics on the slow manifold evolve after a finite amount of slow time. From this reduction we obtain a one-dimensional map dependent on the parameters of the original biophysical equations. In some parameter regimes, one can deduce that the original equations have solutions in which the active phase of the originally excitable cell is constant from burst to burst, while in other parameter regimes it is not. The existence or absence of this kind of regulation corresponds to qualitatively different dynamics in the one-dimensional map. The computations associated with the reduction and the analysis of the dynamics includes the use of coordinates that parameterize by time along trajectories, and "singular Poincaré maps" that combine information about flows along a slow manifold with information about jumps between branches of the slow manifold.
\end{abstract}

Keywords: Central pattern generator - Relative coordination Oscillation - Singular perturbation - Subharmonics

\footnotetext{
* This research partially supported in part by NIMH grant MH47150 and NSF grant DMS9200131.
} 


\section{Introduction}

Networks of voltage-gated conductance equations can be high dimensional, and are often difficult to analyze. One strategy for dealing with this is to place heavy reliance on computer simulations. Another is to invent simple caricatures that capture some of the behavior of the original system. The first strategy can be used to display many interesting phenomena, but is rarely appropriate for understanding why the system behaves as it does. The second can yield many insights, but it is often hard to relate those insights directly to the original system.

An intermediate strategy is to develop techniques to reduce the original system to a much simpler system. By "reduce" we mean find a simpler description, and give the conditions on the original system under which the latter is guaranteed to behave like the simpler one. Thus, the explicit reduction is the bridge connecting the analysis of the simpler system to the behavior of the complicated one.

In this paper, we illustrate that strategy by analyzing a network of two neurons, each described by voltage-gated conductance equations, and connected by mutually inhibitory chemical synapses. The equations are five-dimensional, and we reduce them to a one-dimensional map. In different parameter ranges for the differential equations, the resulting map is shown to have qualitatively different solutions. Thus, we can use the reduction to predict how changes of parameters for the full equations change the behavior of the network.

The motivating example, discussed in Sect. 2, comes from a subnetwork of the crustacean stomatogastric ganglia (STG) $[13,14]$. One of the model cells, labeled PD, is an autonomous oscillator; it represents the PD-AB electrically coupled complex, which is the pacemaker complex of the pyloric network. (The properties of the AB and PD cells alone play no part in the analysis.)

In the absence of interaction, the other cell (LP) is excitable, but not oscillatory. The oscillating cell is described by the Morris-Lecar equations [26], a simple set of voltage-gated conductance equations often used to model the envelope of bursting neurons [29]. In this formulation, spikes are ignored. The excitable cell has an extra current, a hyperpolarization-activated inward current $\left(i_{h}\right)[1,10,11,17,24]$. The behavior to be investigated is "subharmonic coordination": when the excitable cell is hyperpolarized, it bursts once for every $n>1$ bursts of the oscillator. This contrasts with behavior of many other oscillating systems in which, as a parameter is changed, one sees $n: m$ coordination for all pairs of integers $n$ and $m[2,6,9]$. As we will discuss in Sect. 5.2, the $n: 1$ coordination is associated with a kind of timing regulation that is not seen when there is $n: m$ coordination. 
The equations used to describe the motivating network are singularly perturbed (also known as "fast-slow systems"), i.e. they have more than one time scale. There are two fast variables (the voltages of the two cells) and three slower ones. In Sect. 3.1, we review the basic concepts of slow manifolds, manifolds of "knees," singular Poincaré maps and other mathematical ideas needed in the later analysis. These ideas are relevant to the analysis of large classes of singularly perturbed equations. In Sect. 3.2 we restrict to fast-slow cells coupled by models of fast synapses, and describe further mathematical ideas, introduced in [22], for analysis of such systems, including "fast threshold modulation." In Sects. 3.3 and 3.4, we introduce ideas needed to compute derivatives of the singular Poincaré maps; these include coordinates based on time differences, and the "compression" of this distance across fast jumps.

Sections 4 and 5 discuss the reduction of the equations in Sect. 2, and a simpler variation on them, to families of one-dimensional maps; they also describe the behavior of periodic solutions to those maps. Section 4 deals with simpler equations in which the extra $\left(i_{h}\right)$ current is absent from the equations for the excitable cell; these equations are four-dimensional. In this case, the slowest time scale is that of the recovery variable of the excitable cell. We show that the equations can be reduced to a one-dimensional map with a discontinuity. On each branch the slope of the map is positive. In some simple cases, this is shown to correspond to the excitable cell bursting on each cycle of the oscillatory cell, or never bursting. In the more interesting cases, when the recovery time of the excitatory cell is long enough compared to the burst time of the oscillator, the reduction produces families of mappings that have been analyzed by Keener [16]. The latter analysis shows that there is $n: m$ coordination; indeed, as a parameter is changed in such families, one generally gets the well-known "devil's staircase," in which some measure of the coordination pattern (such as the ratio $n /(n+m))$ changes continuously with parameters, yet is constant almost everywhere.

In the equations of Sect. 5, the slowest time scale is not the recovery time of the excitable cell, which is now assumed to be in the same range as the burst time of the oscillator. Instead, the slowest time scale is the rise time of the $i_{h}$ current. The coordination patterns displayed by the maps associated with the full equations are very different in some parameter ranges. Again each map has two branches, with a discontinuity. In some parameter ranges (but not all), one of these branches has a negative slope. As shown in LoFaro [23], such maps can have periodic solutions corresponding to $n: 1$ coordination only, and do not give rise to "devil's staircase" patterns as a parameter is changed. In 
Sects. 4 and 5, the reduction procedure is explicit, so it is possible to compute from the equations the shape of the map, and hence the possible periodic solutions to the original unreduced equations. We note that both kinds of patterns have been seen in related simulations done by Wang and Rinzel [34] and analysis done by Xie et al. [37]. Other related work includes $[4,9,19,20]$.

The equations of Sects. 2 and 5 are five-dimensional, with three slow variables. The natural reduction using fast/slow analysis turns out to be a two-dimensional map. To obtain a one-dimensional map from this, we use further information on the slow time scales to obtain a projection onto a submanifold of the domain of the Poincare map. The slow rise time of $i_{h}$, plus the faster recovery time of the excitable cell, forces the image of the natural two-dimensional Poincaré map to lie close to a curve of "pseudo-critical" points. Still another time scale, the reset time of $i_{h}$ when the voltage is high, determines the behavior of the resulting one-dimensional map. By changing this time scale (but still keeping it slow with respect to fast changes in voltage), it is possible to get either $n: 1$ coordination or the full $n: m$ coordination patterns. We also show in Sect. 5 that when there is only $n: 1$ coordination, the slower cell has constant burst time each time it is active. By contrast, when there is $n: m$ coordination, the burst time can change from one burst to another within the same solution.

The reduction from the two-dimensional map to the one-dimensional map is similar in spirit to the usual fast-slow analysis. However, it does not fit into the usual framework, partly because the variables chosen for each branch of the projected manifold change from branch to branch. Also, there need not be an order of magnitude difference between the speed on the slower projected submanifold and the larger submanifold.

Section 6 contains discussions of the computation and use of singular Poincare maps, and of related work. We also discuss the current mathematics in the context of a more general question about the functional consequences of different kinds of dynamics.

\section{Motivating example}

The data motivating the analysis in Sect. 5 comes from a network of the crustacean nervous system known as the stomatogastric ganglion [13, 14]. Data from two of the cells of this network are shown in Fig. 1 [21]. These two cells (PD and LP) have inhibitory synapses on one another. In normal circumstances, the two cells burst in antiphase [8, 28, 30, 33, 34]. However, when the LP cell is given constant hyperpolarizing 
A

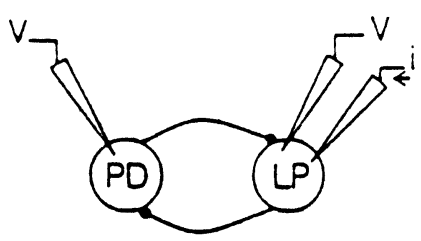

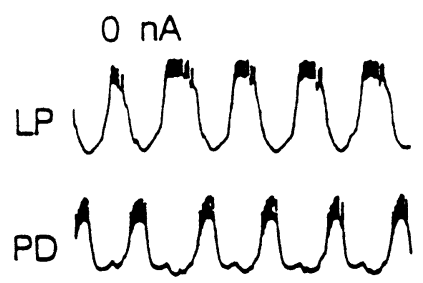

B

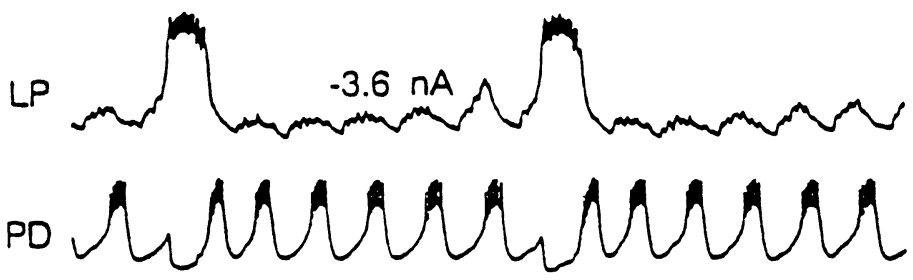

C

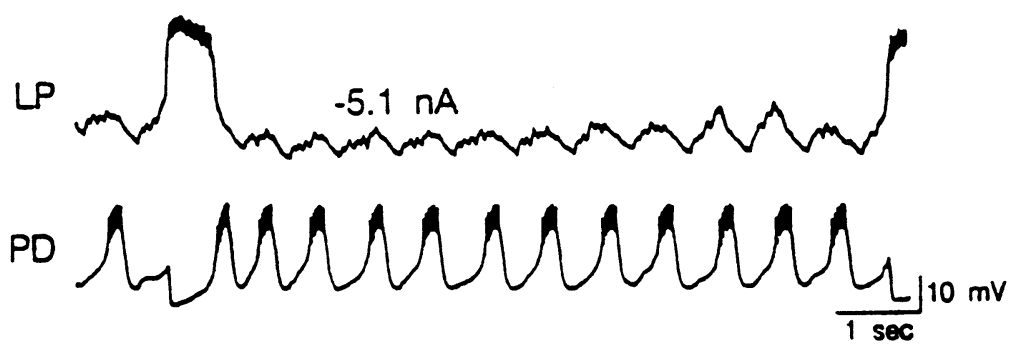

Fig. 1. A: (left) A schematic of the experimental set up. The PD and LP neurons mutually inhibit each other; each neuron's membrane is being monitored intracellularly (V electrodes), and current can be injected into the LP through a second electrode (i electrode). (right) The activity of the two neurons with 0 current injected into the LP neuron. The neurons exhibit 1:1 firing. B: Negative current is injected into the LP neuron until the firing ratio is approximately 6 PD bursts per LP burst. C: Negative current is injected into the LP neuron until the firing ratio is approximately 12 PD bursts per LP burst.

current, the network behavior changes: the LP cell bursts once for every $n \geqq 1$ bursts of the oscillator. The integer $n$ increases with the amount of hyperpolarizing current up to a saturation value.

In a previous publication [21], we reported a simulation that captures this behavior exhibiting only $n: 1$ relative coordination as the hyperpolarization current is varied. These simulations were based on unpublished data by Hooper [15]. Section 5 analyzes a family of equations that includes those used in the simulation of [21]. In this section, we present some of the simulations and a heuristic explanation of how the equations produce subharmonics. However, the heuristic discussion cannot explain why, in some parameter ranges, one gets 


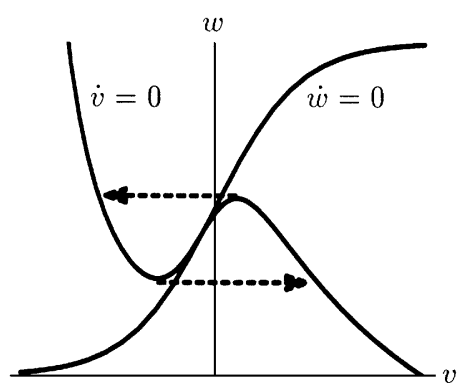

A

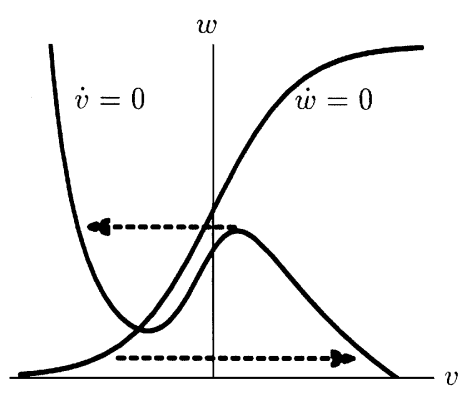

B

Fig. 2. A: The Morris-Lecar equations in the oscillating regime. With the critical point on the middle branch of the $v$-nullcline there exists an asymptotically stable limit cycle. In the singular case $(\varepsilon \ll 1)$ this limit cycle hugs the left and right branches of the $v$-nullcline with "jumps" between these branches occurring at the local extrema. B: The Morris-Lecar equations in the excitable regime. There is an asymptotically stable critical point on the left branch of the $v$-nullcline. Solutions starting to the right of the middle branch or below the minimum of the $v$-nullcline take an excursion around the right branch before approaching the critical point.

only $n: 1$ solutions, while in others one gets the full devil's staircase of all $n: m$ modes of interaction. The analysis of Sect. 5 clarifies this by showing that the different parameter ranges lead to very different one-dimensional maps.

The PD cell is part of an oscillating pacemaker complex, and is represented in the simulations by a two-dimensional relaxation oscillator using the Morris-Lecar equations, which are often used to model the envelope of bursting activity; the high frequency oscillations are not included in this model. Fig. 2A gives a phase-plane portrait of these equations. The LP cell does not burst in the absence of interaction with other cells, and is modeled as an excitable cell. Its equations are again the Morris-Lecar equations, this time chosen so that the cell is excitable rather than oscillatory (see Fig. 2B). In addition, it has an extra ionic current, the hyperpolarization-activated current $i_{h}[1,10,11,17$, 24]. The form of the equations is given in Sect. 5 with details in the Appendix.

The effect of inhibition on both the LP and PD model neurons is the lowering of the $v$-nullcline which prevents the cell from firing until the conclusion of the burst of the opposite cell. In the relevant parameter regime, when the level of $i_{h}$ is small the LP cell cannot fire when released from inhibition, but does fire at higher levels of $i_{h}$. However, while the LP is hyperpolarized the level of $i_{h}$ slowly increases. If there exists a threshold such that at levels of $i_{h}$ past the threshold the LP fires upon release from inhibition, then the LP may fire once after $n$ bursts of the PD. 
This does not guarantee, however, that there will be the same number of PD bursts between LP bursts. To understand why this can occur we must discuss the behavior of $i_{h}$ during LP depolarization. If $i_{h}$ resets to some fixed value during an LP burst then the process described above begins at approximately the same level of $i_{h}$ and hence the same number of PD bursts is required for the level of $i_{h}$ to exceed the threshold. On the other hand, if the level of $i_{h}$ decreases too slowly, we will show that the number of PD bursts between LP bursts (and the length of the LP burst) can be variable within a given trajectory. This will be described in Sect. 5 .

Simulations of this network show that the number of PD bursts between LP bursts increases in steps of 1 as the amount of injected hyperpolarizing current to the LP increases. The reduction to and analysis of the one-dimensional maps show how changing this (or other parameters) can lead to the "period-adding" phenomenon [21]; this is discussed in further detail in Sect. 6.

The results of Sect. 5 also suggest that in the parameter regime where only stable $n: 1$ subharmonics occur there exists intervals of bistability between $n: 1$ regimes and $(n+1): 1$ regimes. This behavior was observed in simulations of the model network in small intervals near the transition between $n: 1$ and $(n+1): 1$ parameter regimes [21].

\section{Mathematical background}

\subsection{Singular Poincaré maps}

We are concerned in this paper with systems of the form

$$
\begin{aligned}
\varepsilon d x / d t=f(x, y), & x \in \mathbf{R}^{n} \\
d y / d t=g(x, y), & y \in \mathbf{R}^{m}
\end{aligned}
$$

or, equivalently,

$$
\begin{aligned}
& d x / d \tau=f(x, y) \\
& d y / d \tau=\varepsilon g(x, y)
\end{aligned}
$$

where $\tau=t / \varepsilon$. For (2) at $\varepsilon=0$, there is a manifold of critical points given by

$$
f(x, y)=0 .
$$

The set of points satisfying (3) is known as the slow manifold. A "submanifold of knees" $\mathscr{M}_{k}$ of (3) is a submanifold on which $\partial f / \partial x$ has a single zero eigenvalue, corresponding to a fold of (3) (see Fig. 3). With 


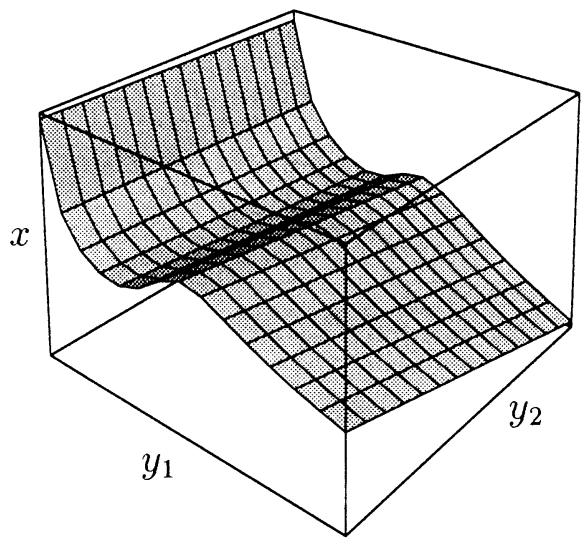

Fig. 3. An example of a slow manifold given by (3). In this example $x \in \mathbf{R}$ and $y \in \mathbf{R}^{2}$. This manifold has exactly two knees; each is one-dimensional.

appropriate non-degeneracy hypotheses, this is a codimension one submanifold of (3).

We shall be interested in singular periodic solutions to (1) or (2). These are unions of solutions to the fast equations

$$
d x / d \tau=f(x, y), \quad y \text { constant }
$$

and the slow equations

$$
d y / d t=g(x(y), y), x(y) \text { satisfying (3). }
$$

We shall assume that along the slow portions of the singular solution, $\partial f / \partial x$ has strictly negative eigenvalues, except where the trajectory hits a manifold of knees. We assume that each slow piece hits such a submanifold transversely. The intersection point is the transition point between the slow segment and the next fast portion.

The fast trajectory joining a slow segment to the next slow segment is a heteroclinic orbit of (4), joining a pair of points satisfying (3). We assume further that for each transition point $p$ on a manifold of knees the $\tau \rightarrow \infty \operatorname{limit} j(p)$ of the heteroclinic orbit from $p$ is a point on (3) for which the eigenvalues of $\partial f / \partial x$ have real parts strictly negative. It follows by continuity that for each nearby point $q$ on the manifold of knees, there is a heteroclinic orbit joining $q$ to a point near $j(p)$.

The Poincaré map of a differential equation is a map from a crosssection of the flow back to itself, using the flow. A singular Poincare map for an equation of the form (1) or (2) is the analogue of that, restricted to the slow manifold. The manifold of knees $\mathscr{M}_{k}$ is codimension one in the slow manifold, and each branch forms a natural cross-section for the flow on the slow manifold. Thus, a singular 
Poincaré map $\mathscr{P}$ is a map from an open set $\mathscr{S}$ on the manifold of knees $\mathscr{M}_{k}$ back to $\mathscr{M}_{k}$, defined on a neighborhood of a point $p_{0}$ on a singular periodic orbit. $\mathscr{P}$ is defined by using (4) and (5) successively, until the last slow trajectory flows to a point near $p_{0}$ in $\mathscr{M}_{k}$. By construction, $p_{0}$ is a fixed point of $\mathscr{P}$.

We say that $p_{0}$ is a stable fixed point of $\mathscr{P}$ if the eigenvalues of $d \mathscr{P}\left(p_{0}\right)$ are strictly less than one in absolute value. If $p_{0}$ is a stable fixed point for $\mathscr{P}$, then it follows from theorems of Mishchenko and Rozov [25] and Bonet [3] that for (1) with $\varepsilon \neq 0$, there is a unique stable periodic orbit near that of $\mathscr{P}$.

\subsection{Coupled oscillatory/excitable systems}

The work of Mishchenko and Rozov and Bonet allows one to reduce the question of the existence and stability of a periodic orbit to the existence and stability of a fixed point of a singular Poincaré map. In this subsection, we introduce the form of the system we will consider in Sects. 4 and 5, including coupling by "fast threshold modulation." The work of this section will imply that there are well-defined singular Poincare maps for the families of equations in Sects. 4 and 5. The stability issue requires more explicit computation, using some ideas applicable only to fast-slow systems; these are introduced in Sects. 3.3 and 3.4.

By an oscillatory/excitable system we shall mean equations of the form

$$
\begin{aligned}
& d v / d \tau=F(v, w), \quad v \in R \\
& d w / d \tau=\varepsilon G(v, w), \quad w \in R^{k} .
\end{aligned}
$$

We require that there be a range of $w$ for which $v \mapsto F(v, w)$ is qualitatively cubic, with two branches (denoted low and high) on which $\partial F / \partial v<0$. There is then a pair of codimension one submanifolds of $F(v, w)=0$ that are local maximum and local minima for $F(v, w)$. These are the manifolds of knees for (6) (see Fig. 3). (If $w$ is scalar, $F(v, w)$ is a curve and the "manifolds" of knees are just a pair of points.) For (6) to be oscillatory, we require that it have a stable singular periodic orbit. For it to be excitable, we require that it have a stable critical point on the low branch and that a sufficiently large perturbation from that point leads to a larger excursion around the high branch before returning to the critical point (see Fig. 2).

We now take a pair of such systems and couple them to get a more complicated system still of the form (2). The coupling is of the form 
called fast threshold modulation (FTM) in [31]. In this kind of coupling, the first (voltage) equation of (6) is replaced by one in which an extra term representing a synaptic current is added. The synaptic current term has the form $\bar{g}(\hat{v})\left(v_{s y n}-v\right)$, where $v_{s y n}$ is a constant (the reversal potential of the synapse), $\hat{v}=\hat{v}(t)$ is the voltage of the presynaptic cell, and $\bar{g}(\hat{v})$ is a sigmoidal function that is zero for $\hat{v}$ sufficiently low and saturates for $\hat{v}$ sufficiently high. We assume that $\bar{g}$ is essentially constant on the low and high branches of $F(\hat{v}, \hat{w})$ (Fig. 4). Thus, the conductance $\bar{g}(\hat{v})$ of the synaptic current depends only on whether the cell is "off" (i.e., on the low branch) or "on", (i.e. on the high branch); it does not depend on the position of the presynaptic cell within a branch. When the presynaptic cell is off, the null-surface of the postsynaptic cell is

$$
F(v, w)=0
$$

when the presynaptic cell is on, the null-surface of the postsynaptic cell is

$$
F(v, w)+\alpha\left(v_{s y n}-v\right)=0
$$

where $\alpha$ is the (approximately) constant value of $\bar{g}(\hat{v})$ on the right branch of the presynaptic cell. For the values of $\alpha$ and $v_{s y n}$ considered here, the extra term in (8) displaces the qualitatively cubic surface in (7) either upward or downward (and changes its shape). An upward shift corresponds to an excitatory current and a downward shift to an inhibitory current. Figure 5 illustrates the inhibitory coupling effect on the postsynaptic cell using the model equations given in the appendix. Note that the added current can change a system from oscillatory to excitatory (as in the case considered here) or vice versa.

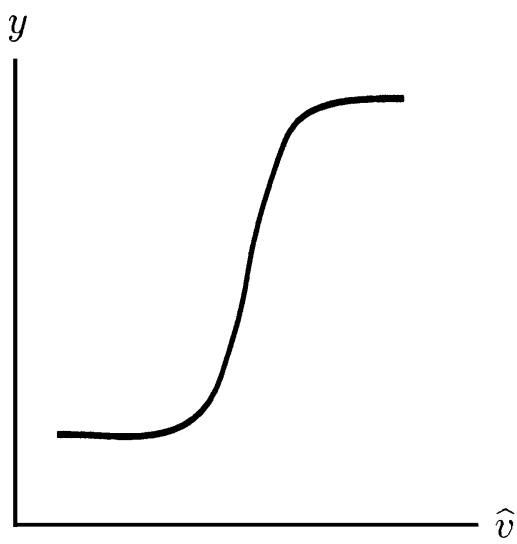

Fig. 4. The graph of $y=\bar{g}(\hat{v}) \cdot \bar{g}$ is assumed to be essentially constant on the low and high branches of $F(\hat{v}, \hat{w})=0$. 


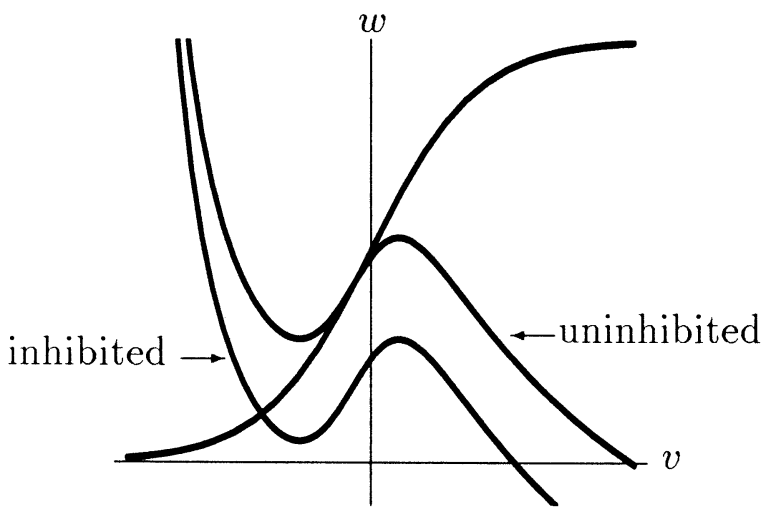

Fig. 5. An example of the inhibited and uninhibited slow manifolds of (7) and (8) using model equations (82) and (83) in the appendix. The effect of inhibition is to lower the $v$-nullcline with a slight change in shape.

Sections 4 and 5 include a discussion of the construction of slow manifolds, manifolds of knees and singular Poincaré maps for oscillatory/excitable systems coupled via FTM. We now go to the mathematical ideas used in those sections in the computation of stability.

\subsection{Time metrics and compression across jumps}

Fast threshold modulation coupling conveys information only at the time of a jump of one of the cells; in between, the cells are essentially uncoupled, with each postsynaptic cell aware only of the branch of its presynaptic cell. Thus, a natural computation of stability of a periodic orbit for the coupled system places special emphasis on the behavior of the coupled system when either cell jumps. For this it is useful to have special coordinate systems in phase space based on time between points. We introduce this coordinate system and its application in a general setting that applies to the model described in Sect. 4. Modifications to this method will be required in the higher dimensional model described in Sect. 5 .

Consider a pair of autonomous scalar differential equations

$$
\begin{aligned}
& \dot{x}_{1}=f_{1}\left(x_{1}\right) \\
& \dot{x}_{2}=f_{2}\left(x_{2}\right)
\end{aligned}
$$

defined on a pair of open intervals $I_{1}$ and $I_{2}$ respectively. If $f_{i}\left(x_{i}\right) \neq 0$ for all $x \in I_{i}, i=1,2$ then each vector field can be used to introduce 
a time-based coordinate system on the appropriate interval. Fix points $p_{i} \in I_{i}$. Then the functions

$$
\tau_{i}\left(x_{i}\right)=\int_{p_{i}}^{x_{i}} \frac{d x_{i}}{f_{i}\left(x_{i}\right)}
$$

are diffeomorphisms from $I_{i}$ to $\mathbf{R}$ that assign to each point a time coordinate dependent on the appropriate differential equation. This time coordinate is simply the time to flow from $p_{i}$ to $x_{i}$.

Let $j: I_{1} \mapsto I_{2}$ be a diffeomorphism such that $x_{2}=j\left(x_{1}\right)$. In the following sections $j$ will be the jump map induced by the fast subsystem that takes a point on one branch of the slow manifold to a point on another branch with the same slow variable coordinates. The time metric allows us to compute changes in "distance" induced by the map $j$. The central notion in that computation is that of "compression across a jump," an idea first used by Somers and Kopell [31]. Consider two points $x_{\alpha} \leqq x_{\beta}$ in $I_{1}$ and their images $j_{s}\left(x_{\alpha}\right)$ and $j_{s}\left(x_{\beta}\right)$ in $I_{2}$. We define the compression across the jump of the interval $\left[x_{\alpha}, x_{\beta}\right]$ as

$$
C_{\alpha \beta}=\frac{\text { time between } j\left(x_{\alpha}\right) \text { and } j\left(x_{\beta}\right)}{\text { time between } x_{\alpha} \text { and } x_{\beta}} .
$$

When the quantity in (12) is less than one in absolute value, the distance (in the time metric) between $x_{\alpha}$ and $x_{\beta}$ decreases across a jump.

The instantaneous compression at $x_{\alpha}$ is

$$
\lim _{x_{\beta} \rightarrow x_{\alpha}} C_{\alpha \beta} \equiv C\left(x_{\alpha}\right) \text {. }
$$

Letting $x_{\beta}=x_{\alpha}+h$ we can rewrite (12) using (11) as

$$
C_{\alpha \beta}=\frac{\int_{j\left(x_{\alpha}\right)}^{j\left(x_{\alpha}+h\right)}\left[f_{2}\left(x_{2}\right)\right]^{-1} d x_{2}}{\int_{x_{\alpha}}^{x_{\alpha}+h}\left[f_{1}\left(x_{1}\right)\right]^{-1} d x_{1}} .
$$

Taking the limit as $h \rightarrow 0$ gives

$$
C\left(x_{\alpha}\right)=\frac{f_{1}\left(x_{\alpha}\right)}{f_{2}\left(j\left(x_{\alpha}\right)\right)} j^{\prime}\left(x_{\alpha}\right) .
$$

Thus the instantaneous compression ratio is the value of the vector field at the jump-off point divided by the value of the vector field at the jump-on point times the derivative of the diffeomorphism $j$.

In the applications described in this paper, the diffeomorphism $j$ is described by the fast equations (4) and is therefore the identity function when expressed as a function of $x$. In this case the instantaneous compression ratio is given by

$$
C\left(x_{\alpha}\right)=\frac{f_{1}\left(x_{\alpha}\right)}{f_{2}\left(j\left(x_{\alpha}\right)\right)} .
$$




\subsection{Compression and fast-slow systems}

The compression calculations we use in this paper are easiest to describe when $w$ is scalar for each of the oscillatory/excitable systems. The coupled system is then four-dimensional, with a two-dimensional slow manifold. The manifolds of knees in the singular system are curves, with one cell at a local maximum or minimum and the other cell away from such a point.

We shall denote the high ("on") and low ("off") branches of the cubic nullclines in the absence of inhibition by $H$ and $L$. Subscripts on $H$ and $L$ denote the cell. The same notation with a hat on the $H$ or $L$ denotes nullclines for the inhibited cells. Thus, for example, $\hat{L}_{2}$ denotes the low branch of cell 2 when the latter is receiving inhibition from cell 1 .

The distance between two points will be defined only for points on the same branch of the curve of knees. For ease of exposition, we specify that the coupling is inhibitory and choose a particular branch of the curve of knees. Assume, for example, that cell 2 is at its local maximum and cell 1 is on the low branch of its inhibited cubic given by (8) (see Fig. 6). Let $p_{\alpha}$ and $p_{\beta}$ denote points on $\hat{L}_{1}$ and $j\left(p_{\alpha}\right)$ and $j\left(p_{\beta}\right)$ points on $H_{1}$ having the same $w_{1}$ coordinates as $p_{\alpha}$ and $p_{\beta}$. When both $\hat{L}_{1}$ and $H_{1}$ are parameterized using $w_{1}$ then the function $j$ is the identity function. The distance between $p_{\alpha}$ and $p_{\beta}$ is defined to be the time for a singular trajectory of cell 1 to go between the cell 1 components of the two points. This time can be determined from the rescaled (6) and
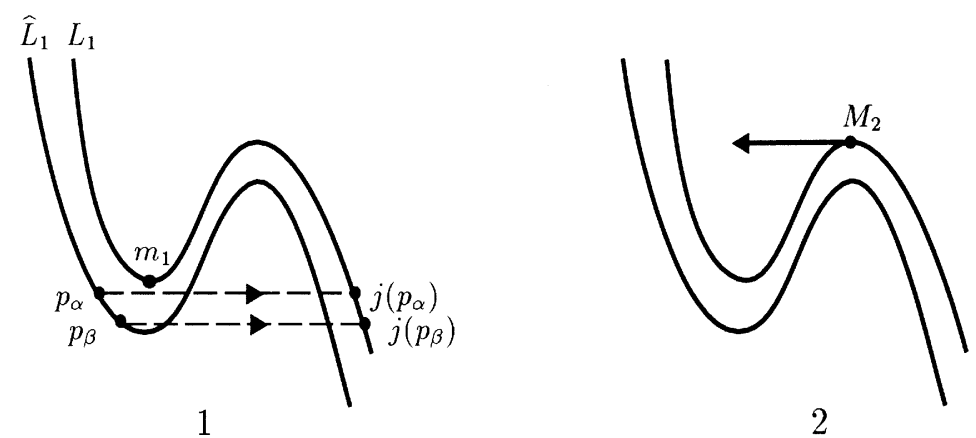

Fig. 6. Compression across a jump. The points $\left(p_{\alpha}, M_{2}\right)$ and $\left(p_{\beta}, M_{2}\right)$ are on a knee of the slow manifold. The time-distance between these points is the time to flow from $p_{\alpha}$ to $p_{\beta}$. If we assume $p_{\alpha}$ and $p_{\beta}$ to be below the threshold $m_{1}$ then the jump from $M_{2}$ causes $p_{\alpha}$ and $p_{\beta}$ to jump to the points $j\left(p_{\alpha}\right)$ and $j\left(p_{\beta}\right)$ on the branch $H_{1}$. The time distance between these points is the time to flow from $j\left(p_{\beta}\right)$ to $j\left(p_{\alpha}\right)$. The compression ratio is the time from $j\left(p_{\beta}\right)$ to $j\left(p_{\alpha}\right)$ divided by the time from $p_{\alpha}$ to $p_{\beta}$. 
satisfies

$$
\frac{d w_{1}}{d t}=G_{1}\left(\hat{v}\left(w_{1}\right), w_{1}\right)
$$

where $v_{1}$ and $w_{1}$ are the coordinates of cell 1 and $\hat{v}\left(w_{1}\right)$ is the parameterization of the low branch $\hat{L}_{1}$ of (8) in terms of $w_{1}$. Similarly, the time from $j\left(p_{\beta}\right)$ to $j\left(p_{\alpha}\right)$ can also be determined from the rescaled (6) and satisfies

$$
\frac{d w_{1}}{d t}=G_{1}\left(v\left(w_{1}\right), w_{1}\right)
$$

where $v\left(w_{1}\right)$ parameterizes the branch $H_{1}$ by $w_{1}$. By applying the ideas of Sect. 3.3 we compute the instantaneous compression ratio at $p_{\alpha}$ in the jump from $\hat{L}_{1}$ to $H_{1}$ to be

$$
C_{\hat{L}_{1} H_{1}}\left(p_{\alpha}\right)=\frac{G_{1}\left(\hat{v}\left(w_{1}\right), w_{1}\right)}{G_{1}\left(v\left(w_{1}\right), w_{1}\right)} .
$$

In other words, the instantaneous compression ratio $C\left(p_{\alpha}\right)$ is simply the ratio of the singular vector field evaluated at the jump-off point to the singular vector field evaluated at the jump-on point. This notation is indicative of the notation used in all compression calculations; subscripts indicate the jump-off and jump-on branches, in that order.

In Sect. 4 we will compute the derivative of the singular Poincare map by factoring it into maps between branches of the curves of knees, and computing the derivative of each factor in terms of compression across jumps. The computation in Sect. 5 is more elaborate because of the higher dimension involved, and we leave till that section the extra structure that will be needed.

\section{Complex dynamics due to slow recovery of the excitable cell}

In this section we analyze a simpler set of equations than the ones given in the motivating example of Sect. 2. In this simpler example, there is no $i_{h}$ current, and the equations are four-dimensional rather than five-dimensional. The motivation for this section is two-fold: first, we use it to introduce some concepts and techniques. Secondly, we wish to contrast the mechanisms that produce complex dynamics in this case with the mechanisms that produce the dynamics introduced in Sect. 2 and analyzed below in Sect. 5 .

There are four subsections of this section. The first introduces the equations and the hypotheses. The second describes the slow manifolds and manifolds of knees, and defines the singular Poincare map 
associated with these equations. The singular Poincare map is shown to be a one-dimensional map with a discontinuity, such that each of the two parts has positive slope. In the third subsection we give the possible maps that satisfy those restrictions, and discuss the kinds of periodic solutions such maps can have. In the final subsection, we show how to compute the associated one-dimensional map from a given set of equations. This last step provides the connection between the biophysical properties embodied in the parameters of the equations and the resulting behavior of the system.

\subsection{Equations and hypotheses}

The four-dimensional equations, like the equations in Sect. 2, describe an oscillator and an excitable cell coupled by mutual inhibition. The major difference is that the $i_{h}$ current is removed in cell 1 , the excitable cell. The full equations are

$$
\begin{aligned}
d v_{1} / d \tau & =F_{1}\left(v_{1}, w_{1} ; I_{1}\right)+\bar{g}\left(v_{2}\right)\left(v_{s y n}-v_{1}\right) \\
d w_{1} / d \tau & =\varepsilon G_{1}\left(v_{1}, w_{1}\right) \\
d v_{2} / d \tau & =F_{2}\left(v_{2}, w_{2} ; I_{2}\right)+\bar{g}\left(v_{1}\right)\left(v_{s y n}-v_{2}\right) \\
d w_{2} / d \tau & =\varepsilon G_{2}\left(v_{2}, w_{2}\right)
\end{aligned}
$$

These have the form of the specific equations given in the Appendix, with the $h$-current set to zero. We assume that (20) satisfies the following hypotheses.

H1: For the fast $v_{1}$ and $v_{2}$ equations of (20), we assume that there are four sets of stable critical points, each two-dimensional, corresponding to high or low branch for each cell. This is easily verified for the equations given in the appendix.

H2: In the absence of inhibition, cell 1 has a stable critical point on the unexcited branch $L_{1}$ and cell 2 has an unstable critical point on the middle branch. The parameter $v_{s y n}$ and the function $\bar{g}$ are chosen so that both cells have stable critical points on branch $\hat{L}_{i}, i=1,2$ in the presence of inhibition. With these choices the nullclines for the uninhibited and inhibited cells are as in Fig. 7.

H3: The signs of $G_{1}$ and $G_{2}$ are assumed to be such that $w_{i}$ decreases above $G_{i}\left(v_{i}, w_{i}\right)=0$ and increases below it. This implies that cell 2 is an oscillator in the absence of inhibition. 


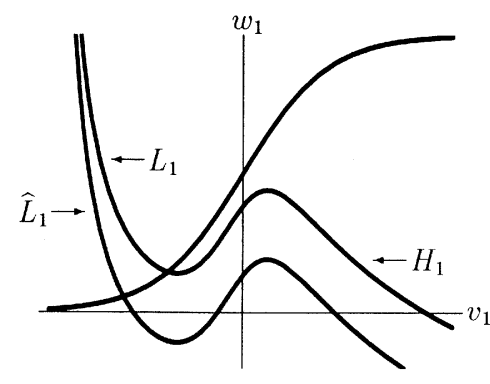

1

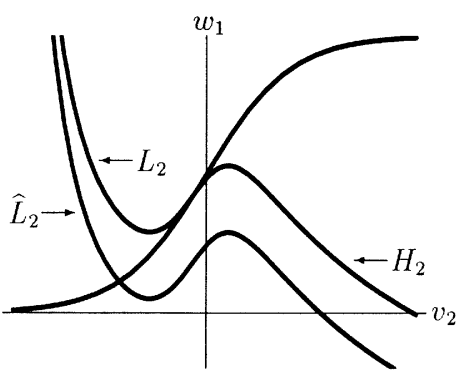

2

Fig. 7. The nullclines for cells 1 and 2 in the uninhibited and inhibited states. The stable branches of each uninhibited cell are labeled $L_{i}$ and $H_{i}$. The stable inhibited "low" branches are labeled $\hat{L}_{i}$.

H4: When cell 1 fires, it fires for a long enough period of time that cell 2 can recover from its previous firing. Thus, cell 2 can fire immediately upon release of inhibition from cell 1 . Geometrically, this means that the cell 2 singular trajectory is on $\hat{L}_{2}$ and below the minimum of the uninhibited cell 2 nullcline at the end of cell 1 firing (see Fig. 7). We make no such assumptions on the relationship between the cell 2 firing time and the cell 1 recovery time. Indeed, as will be shown below, the recovery time of cell 1 is one of the important determinants of the network behavior; to produce the most complicated behavior to be described below, this time cannot be too short.

\subsection{The singular Poincaré map}

In Sect. 3, we discussed the general notion of slow manifolds, manifolds of knees and singular Poincaré maps. We now say what these objects are for (20).

We denote the four sets of stable critical points described in $\mathrm{H} 1$ by $H H, H L, L H$ and $L L$. For example, $H L=\left\{\left(p_{1}, p_{2}\right) \mid p_{1} \in H_{1}, p_{2} \in \hat{L}_{2}\right\}$. (The hats are not used in this notation but are implicit: if cell 1 is on its high branch, cell 2 is receiving inhibition, whether cell 2 is on its high or low branch.) The other three sets are defined analogously. These are the slow manifolds of (20) and are illustrated in Fig. 8A.

Of the above four sets, only $H L, L H$, and $L L$ are of interest for periodic solutions. The reason is that trajectories of the fast equations never jump to the $H H$ set from another of the sets; thus the $H H$ set is never part of a singular periodic trajectory. On each of the $H L, L H$, and $L L$ slow manifolds, there is a curve which is a branch of the manifold of knees. 

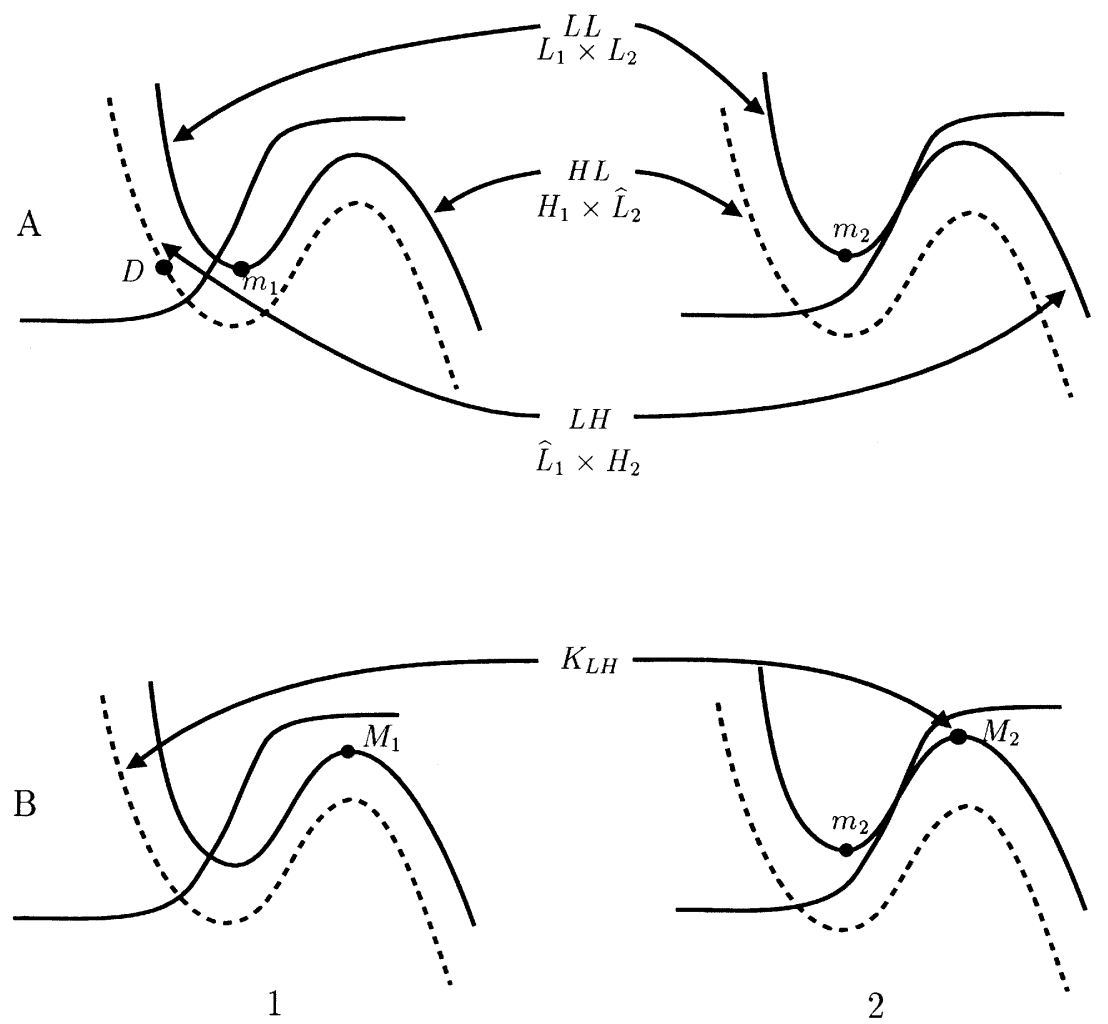

Fig. 8. A: The stable branches of the slow manifold of (15). Each is the Cartesian product of a stable branch of cell 1 (either inhibited or uninhibited) and a stable branch of cell 2 . The point $D$ is the point on $\hat{L}_{1}$ with the same $w_{1}$-coordinate as the minimum $m_{1}$ on the uninhibited nullcline $L_{1}$. B: The knee of branch $L H$ (denoted $K_{L H}$ ) is the product of $\hat{L}_{1}$ and the point $M_{2}$.

1. For $L L$, the curve is $\left\{\left(p_{1}, p_{2}\right) \mid p_{1} \in L_{1}, p_{2}=\min \left(L_{2}\right) \equiv m_{2}\right\}$.

2. For $H L$, the curve is $\left\{\left(p_{1}, p_{2}\right) \mid p_{1}=\max \left(H_{1}\right) \equiv M_{1}, p_{2} \in \hat{L}_{2}\right.$ and $p_{2}$ below $\left.m_{2}\right\}$.

3. For $L H$, the curve is $\left\{\left(p_{1}, p_{2}\right) \mid p_{1} \in \hat{L}_{1}, p_{2}=\max H_{2} \equiv M_{2}\right\}$.

The knee of branch $L H$ is illustrated in Fig. 8B and is denoted $K_{L H}$ there. Any trajectory starting at a point on $L L, L H$ or $H L$ must reach the appropriate curve of knees in finite (slow) time, following equations (20). This is a consequence of the fact that the branches $L_{1}$, $\hat{L}_{1}$, and $\hat{L}_{2}$ each have a critical point that prevents motion of one of the cells beyond it (see Figs. 8A, B). Thus other knees of each branch which lie beyond these critical points can be ignored. In item 2 above the restriction that $p_{2}$ be below the minimum of $L_{2}$ in the knee $H L$ comes 
from hypothesis $\mathrm{H} 4$; this implies that $p_{2}$ is below $m_{2}$ by the time $p_{1}$ reaches $M_{1}$.

As explained in Sect. 3, upon reaching a point on the manifold of knees, a singular trajectory jumps according to the fast equations to another piece of the slow manifold. The following proposition summarizes the destinations of the jumps from the manifolds of knees.

Proposition 4.1. 1. The fast trajectory from a point on the LL branch of knees tends to a point on the LH slow manifold.

2. A point on the HL branch of knees also goes to a point on the $L H$ slow manifold.

3. On the LH branch of knees, the jump is a discontinuous map: for $p_{1} \in \hat{L}_{1}$ and above the minimum of $L_{1}\left(m_{1}\right)$, the trajectory jumps to $L L ;$ for $p_{1}$ below $m_{1}$, the trajectory jumps to $H L$.

Proof. Part 1 is immediate from Figs. 8A, B: upon reaching the minimum of $L_{2}$, cell 2 fires. Assertion 2 follows from the hypothesis that cell 2 recovers quickly enough from inhibition. Thus, when cell 1 stops firing and releases cell 2 from inhibition, cell 2 fires. Assertion 3 is also immediate from Figs. 8A, B: when cell 1 is released from inhibition, its destination depends on whether its upward jump is impeded by the $L_{1}$ nullcline.

Each curve of knees is a cross-section of the slow manifold, and can be used to define a singular Poincaré map. In our case, an especially convenient cross-section is the $L H$ curve of knees, for which cell 2 is at $M_{2}$, the max of $H_{2}$. The Poincare map $\mathscr{P}$ defined on the $L H$ curve of knees, takes a point $p_{1} \in \hat{L}_{1}$ to another point on $\hat{L}_{1}$. Starting from the point $\left(p_{1}, M_{2}\right)$ the trajectory undergoes a sequence of fast jumps and slow flows until cell 2 reaches $M_{2}$ again. It follows from Proposition 4.1 and $\mathrm{H} 3$ that this singular orbit must return to the knee of $L H$. The map $\mathscr{P}$ is discontinuous, with a discontinuity at $D$, the point in $\hat{L}_{1}$ at the same height as the minimum of $L_{1}$ (see Figs. 9 and 10). For ease of notation, we drop the subscript 1 on $p_{1}$ when describing a point in the domain of $\mathscr{P}$. We let $\mathscr{P}^{-}$denote $\mathscr{P}$ for points $p$ above $D$ and $\mathscr{P}^{+}$denote $\mathscr{P}$ for points below $D$. For $p=D$, we consider $\mathscr{P}$ to be doubly defined as the limits of $\mathscr{P}^{-}$and $\mathscr{P}^{+}$as $p \rightarrow D$.

Figure 9 illustrates the construction of $\mathscr{P}^{-}$. Consider the initial condition $\left(p, M_{2}\right)$ on the $L H$ knee. Because $p$ is above the threshold for firing, upon release from inhibition $p$ jumps to the point marked $j(p)$ and $M_{2}$ to the point marked $j\left(M_{2}\right)$. The point $\left(j(p), j\left(M_{2}\right)\right)$ is on branch $L L$. These points then flow under the slow system until reaching the knee of $L L$ when cell 2 reaches the point $m_{2}$. Note that the critical point on $L_{1}$ prevents cell 1 from reaching the knee $m_{1}$. We denote the state of 


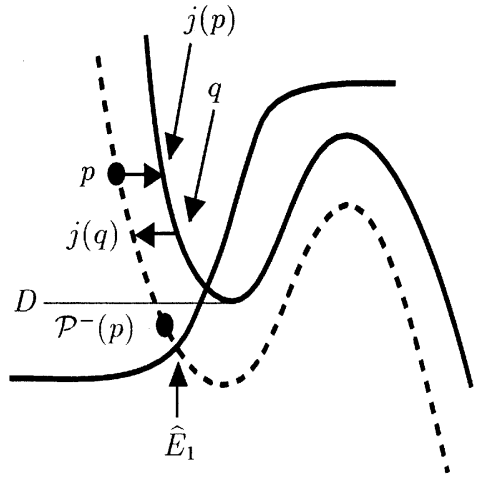

1

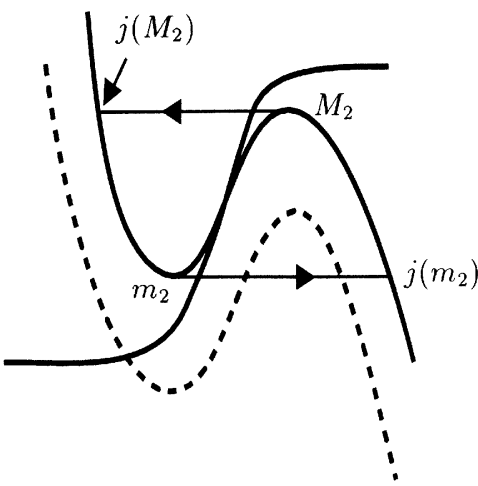

2

Fig. 9. A graphical representation of the map of knees $\mathscr{P}^{-}$. Because $p$ is above $D$, The first jump takes $p$ to $j(p)$ on $L_{1}$. The first flow maps this point to $q$ which in turn jumps back to $\hat{L}_{1}$ and finally flows to the point $\mathscr{P}^{-}(p)$.

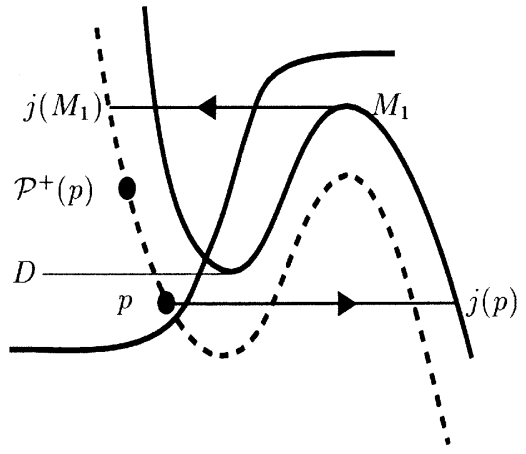

1

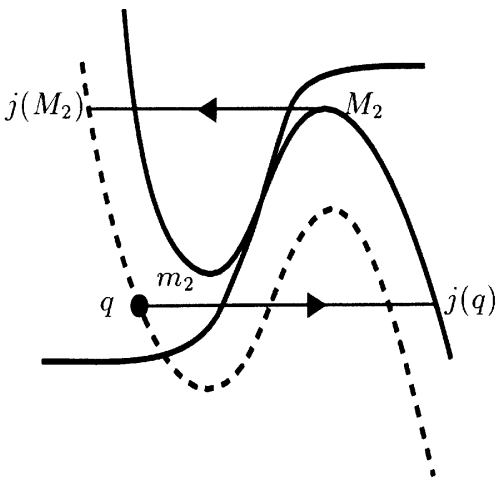

2

Fig. 10. A graphical representation of the map of knees $\mathscr{P}^{+}$. Because $p$ is below $D$, the first jump takes $p$ to $j(p)$ on $H_{1}$ and $M_{2}$ to $j\left(M_{2}\right)$ on $\hat{L}_{2}$. The first flow maps these points to $M_{1}$ and $q$ respectively. The second jumps takes $M_{1}$ to $j\left(M_{1}\right)$ on $\hat{L}_{1}$ and $q$ to $j(q)$ on $H_{2}$. They then flow until $j(q)$ reaches $M_{2}$. The corresponding point on $\hat{L}_{1}$ is $\mathscr{P}^{+}(p)$.

cell 1 by $q$ in Fig. 9. Another jump now occurs, this time to branch $L H$ and the point $\left(j(q), j\left(m_{2}\right)\right)$. Finally, the system flows until cell 2 returns to $M_{2}$. The value of cell 1 at this point is $\mathscr{P}^{-}(p)$.

Figure 10 illustrates the case where $p$ is below the threshold. This time the system jumps to branch $H L$ with cell 1 inhibiting cell 2 . The system flows until $j(p)$ reaches $M_{1}$ and hence the knee of $H L$. At this point cell 2 is at the point $q$ on $\hat{L}_{2}$ which is below the threshold by assumption $\mathrm{H} 4$. Thus the next jump takes the system to branch $L H$ on 
which it flows until cell 2 returns to $M_{2}$. The value of cell 1 at this point is $\mathscr{P}^{+}(p)$.

Remark 4.1. Assumption H4 simplifies the description of the singular Poincare map $\mathscr{P} . \mathscr{P}$ is still well-defined if this assumption is not made. However, derivative calculations used in subsequent sections to describe dynamical properties of the model are no longer valid. In the notation given above, this assumption states that the time to flow from j $\left(\mathrm{M}_{2}\right)$ to $j\left(m_{2}\right)$ on $\hat{L}_{2}$ is short relative to the burst length of cell 1 . Numerical calculations using the model equations given in the appendix suggest that this assumption is reasonable.

\subsection{Possible behavior of the map $\mathscr{P}$}

We shall now show that both $\mathscr{P}^{+}$and $\mathscr{P}^{-}$are non-decreasing (orientation preserving). (The work in Sect. 4.4 implies the stronger result that the derivatives of $\mathscr{P}^{+}$and $\mathscr{P}^{-}$are strictly positive.) We then present a catalogue of possible one-dimensional maps that are piecewise increasing, and describe some of the behavior of each of them. Our convention is that increasing $p$ means increasing values of the $w_{1}$ component of $p$.

We analyze separately the functions $\mathscr{P}^{+}$and $\mathscr{P}^{-}$, again using Figs. 9 and 10. Now consider $\mathscr{P}^{-}$, and let $p_{\alpha}$ and $p_{\beta}$ be two points in its domain with $p_{\alpha}>p_{\beta}$. The trajectories of each of those initial points are as in Fig. 9, with two jumps, and two flows. Note that each jump and each flow is order preserving. Thus $\mathscr{P}^{-}\left(p_{\alpha}\right)>\mathscr{P}^{-}\left(p_{\beta}\right)$, so $d \mathscr{P}^{-} / d p \geqq 0$.

We now consider $p_{\alpha}>p_{\beta}$ in the domain of $\mathscr{P}^{+}$. Each of the two associated trajectories is as in Fig. 10, again with two jumps and two flows. The images $j\left(p_{\alpha}\right), j\left(p_{\beta}\right) \in H_{1}$ after the first jump satisfy $j\left(p_{\alpha}\right)>j\left(p_{\beta}\right)$, i.e., $j\left(p_{\alpha}\right)$ has a larger $w_{1}$-component. This implies that $\tau_{1}\left(p_{\alpha}\right)<\tau_{1}\left(p_{\beta}\right)$, where $\tau_{1}\left(p_{\alpha}\right)$ (respectively $\left.\tau_{1}\left(p_{\beta}\right)\right)$ is the time for cell 1 to flow from $j\left(p_{\alpha}\right)$ (respectively $j\left(p_{\beta}\right)$ ) to $M_{1}$. Thus, the trajectory of cell 2 corresponding to initial point point $p_{\beta}$ for cell 1 has a longer time of flow along $\hat{L}_{2}$ while cell 1 is excited than the trajectory corresponding to $p_{\alpha}$. This implies that when cell 1 reaches $M_{1}$, the positions $q_{\alpha}$ and $q_{\beta}$ on $\hat{L}_{2}$ satisfy $q_{\alpha}>q_{\beta}$. The second jump is initiated when cell 1 reaches $M_{1}$, so the positions of the two trajectories on $H_{2}$ after the second jump satisfies $j\left(q_{\alpha}\right)>j\left(q_{\beta}\right)$. Hence, the time $\tau_{2}\left(p_{\alpha}\right)$ from $j\left(q_{\alpha}\right)$ to $M_{2}$ is shorter than the time $\tau_{2}\left(p_{\beta}\right)$ from $j\left(q_{\beta}\right)$ to $M_{2}$, giving cell 1 a longer time to flow from $j\left(M_{1}\right)$ on $\hat{L}_{1}$ in case $\beta$ than in case $\alpha$. The resulting image, after two jumps and two flows, is by definition $\mathscr{P}^{+}$and the above shows that $\mathscr{P}^{+}\left(p_{\alpha}\right)>\mathscr{P}^{+}\left(p_{\beta}\right)$, so $d \mathscr{P}^{+} / d p \geqq 0$. 
We now describe some of the behavior of discontinuous maps both pieces of which have a positive derivative. We divide up our description into cases.

I. Simplest: $\mathscr{P}^{-}(D)>\mathscr{P}^{+}(D)$. In this case, there is always a fixed point for $\mathscr{P}$, and sometimes more than one. In Fig. $11 \mathrm{~A}, \mathscr{P}^{-}$has a fixed point and $\mathscr{P}^{+}$does not. For each $p \in \mathscr{P}^{+}$there exists a positive integer $n$ such that $\left(\mathscr{P}^{+}\right)^{n}(p)$ lies in the domain of $\mathscr{P}^{-}$. A fixed point of $\mathscr{P}^{-}$ corresponds to a periodic orbit in which cell 1 never fires. All initial
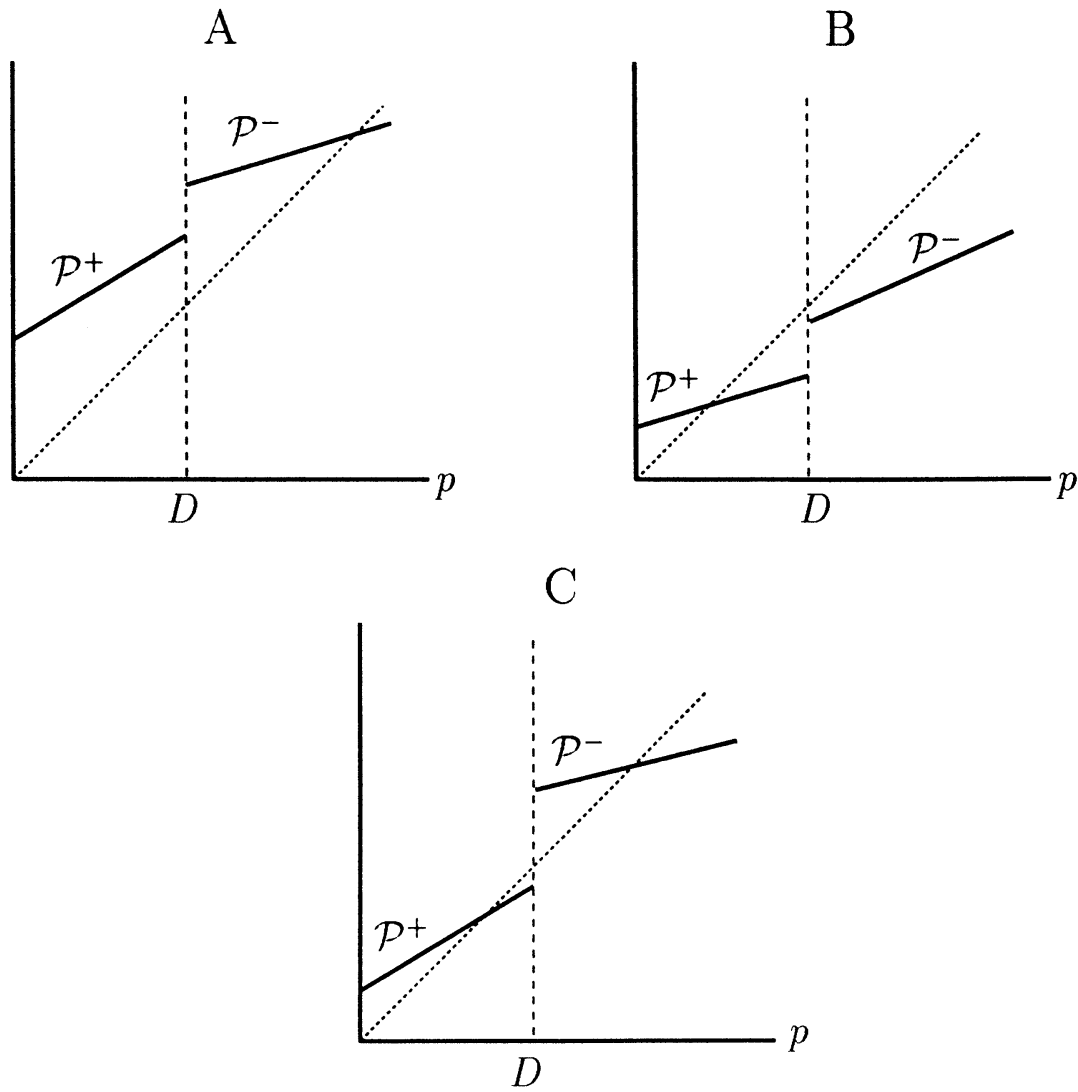

Fig. 11. Behaviors of $\mathscr{P}$ when $\mathscr{P}^{+}(D)<\mathscr{P}^{-}(D)$ (case I). A: There exists at least one fixed point in the domain of $\mathscr{P}^{-}$and all points in the domain of $\mathscr{P}$ are attracted to one of these fixed points. This corresponds to $0: 1$ firing and small amplitude oscillations of cell 1. B: There exists at least one fixed point in the domain of $\mathscr{P}^{+}$and all points in the domain of $\mathscr{P}$ are attracted to one of these fixed points. This corresponds to $1: 1$ firing of cell 1 and cell 2. C: Both $\mathscr{P}^{-}$and $\mathscr{P}^{+}$have attracting fixed points. The domain of $\mathscr{P}^{-}$is mapped into itself and the domain of $\mathscr{P}^{+}$is mapped into itself. This corresponds to a bistability where, depending on the initial condition the model, exhibits either $0: 1$ or $1: 1$ firing. 
conditions tend to a fixed point of $\mathscr{P}^{-}$and hence to this state. This corresponds to small amplitude oscillations of cell 1. In Fig. 11B, $\mathscr{P}^{+}$has a fixed point and $\mathscr{P}^{-}$does not. A fixed point of $\mathscr{P}^{+}$corresponds to a periodic orbit in which cell 1 fires in every cycle of the cell 2 oscillation. All points in the domain of $\mathscr{P}^{-}$are eventually iterated into the domain of $\mathscr{P}^{+}$. In Fig. $11 \mathrm{C}$, both $\mathscr{P}^{+}$and $\mathscr{P}^{-}$have fixed points corresponding to no firing or firing on every cycle; the limiting behavior of a given trajectory then depends on initial conditions. The fixed points in cases (A)-(C) are stable if the slope of $\mathscr{P}$ at that point is less than one in absolute value.

II. More interesting: $\mathscr{P}^{-}(D)<\mathscr{P}^{+}(D)$. As in case I, it is possible to have fixed points for $\mathscr{P}^{+}$and $\mathscr{P}^{-}$corresponding to no firing or firing on every cycle (see Figs. 12A, B). In the more interesting sub-case, neither branch has a fixed point (Fig. 12C). We focus on this case, which itself can have different behaviors.

All points in the domain of $\mathscr{P}$ are eventually iterated into the intersection of the images of $\mathscr{P}^{+}$and $\mathscr{P}^{-}$. This interval $J$ is forward invariant and we can restrict our analysis to $J$. The map $\mathscr{P}$ restricted to $J$ is as in Fig. 13A or B.

The cases in Figs. 13A and B correspond to the "no-overlap" and "overlap" cases. Both of these have been analyzed by Keener [16]. In the no-overlap case, the behavior is very similar to that of continuous circle maps, with the existence of periodic points determined by a rotation number $n /(n+m)$ ( $n$ and $m$ positive integers) that varies continuously with parameters [22]. A rotation number of $n /(n+m)$ corresponds to a periodic orbit with $n$ iterates of $\mathscr{P}^{-}$and $m$ iterates of $\mathscr{P}^{+}$. In other words, cell 1 fires $m$ times for every $n+m$ oscillations of cell 2 . As some parameter (such as the point of discontinuity) is changed, the graph of rotation number versus that parameter forms a "devil's staircase" [5], hitting all rational rotation numbers between any two in the range of that function. (The point of discontinuity can be varied by changing the amount of inhibitory current $I$ injected into cell 2.) We will see that the map derived in Sect. 5 can have very different behavior. The overlap case has potentially more complicated behavior, including chaotic trajectories. We note that if $\left|d \mathscr{P}^{ \pm} / d p\right| \leqq 1$, the overlap is automatically ruled out.

\subsection{Detailed neuronal behavior and the choices of $\mathscr{P}$}

In this section, we discuss what properties of the original equations (20) determine if $\mathscr{P}$ is in case I or case II, and compute $d \mathscr{P}^{ \pm} / d p$ where the 
A

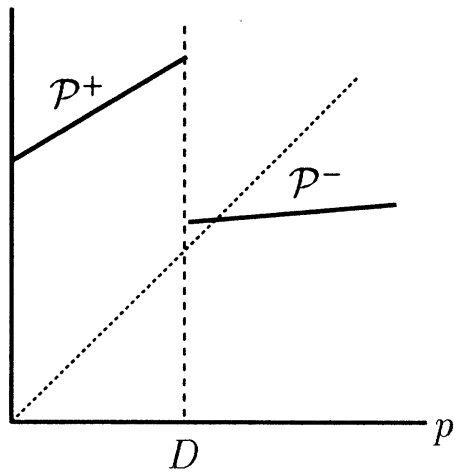

B

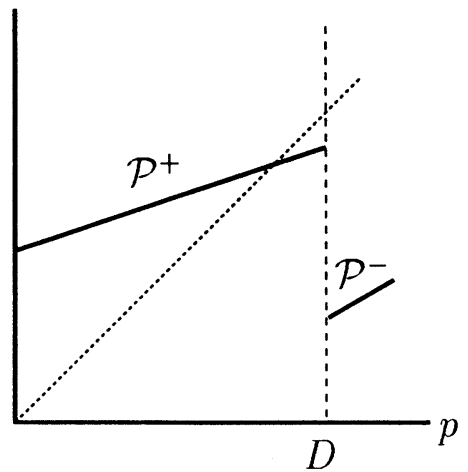

C

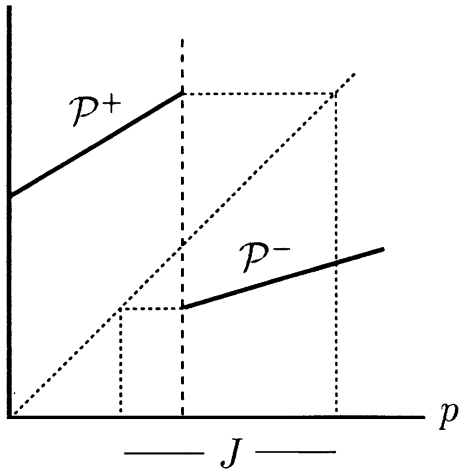

Fig. 12. Behaviors of $\mathscr{P}$ when $\mathscr{P}^{-}(D)<\mathscr{P}^{+}(D)$ (case II). A: There exists a fixed point in the domain of $\mathscr{P}^{-}$and all points in the domain of $\mathscr{P}$ are attracted to this fixed point. This corresponds to no firing of cell 1 . B: There exists a fixed point in the domain of $\mathscr{P}^{+}$and all points in the domain of $\mathscr{P}$ are attracted to this fixed point. This corresponds to 1: 1 firing of cell 1 and cell 2. C: Neither $\mathscr{P}^{-}$nor $\mathscr{P}^{+}$possess fixed points. All points are eventually mapped into the interval $J$ which is forward invariant.

coordinates of $p$ are the time coordinates introduced in Sect. 3.3. From the latter, we can find conditions under which there are stable periodic points. These results, unlike the others in this section, depend on further information about the slow equations of (20).

We first factor each of $\mathscr{P}^{-}$and $\mathscr{P}^{+}$into a pair of one-dimensional maps; $\mathscr{P}^{-}=f_{2} \circ f_{1}$ and $\mathscr{P}^{+}=g_{2} \circ g_{1}$. We start with $\mathscr{P}^{-}$and use the fact that the trajectory of cell 2 over one cycle is not perturbed by the firing of cell 1; hence it is determined independent of the initial position of cell 1 (see Fig. 14). Let $f_{1}$ be the first jump and flow, and $f_{2}$ be the second jump and flow. $f_{1}$ (respectively $f_{2}$ ) has domain in $\hat{L}_{1}$ (respectively $L_{1}$ ) and maps into $L_{1}$ (respectively $\hat{L}_{1}$ ). (Note that these domains can be 

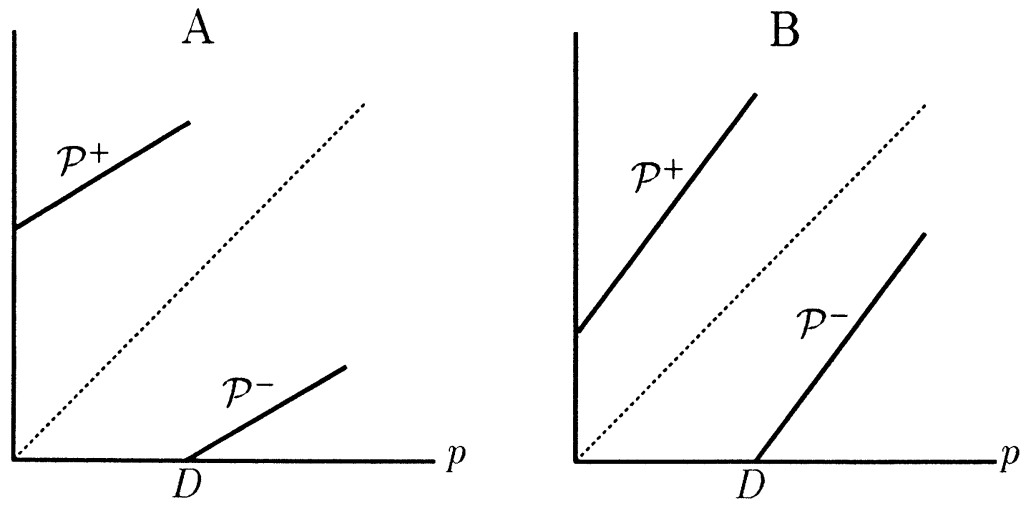

Fig. 13. A: The non-overlapping regime of case II. The map $\mathscr{P}$ has a well-defined rotation number independent of initial condition. A rational rotation number of $n /(n+m)$ corresponds to $m$ firings of cell 1 for every $n+m$ firings of cell 2 . B: The overlapping case. In this scenario more complex dynamics can occur.

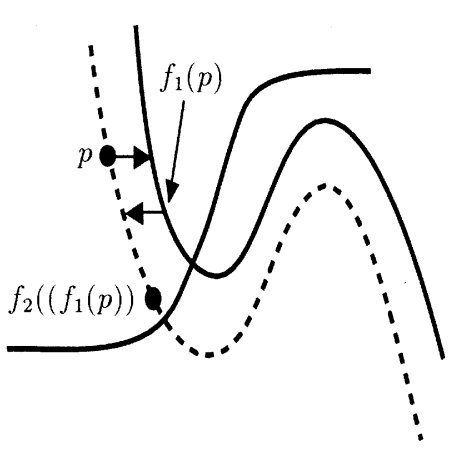

1

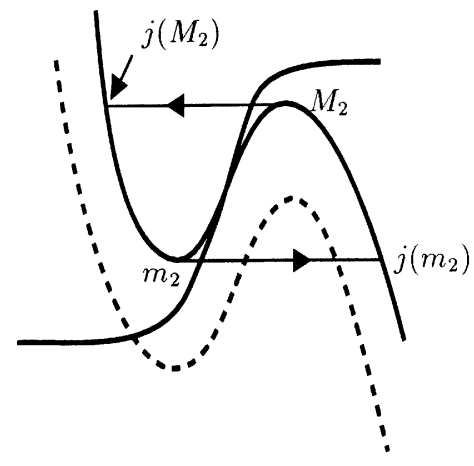

2

Fig. 14. The functions $f_{1}$ and $f_{2} . f_{1}$ maps $p \in \hat{L}_{1}$ to $f_{1}(p) \in L_{1} . f_{2}$ maps $q \in L_{1}$ to $f_{2}(q) \in \hat{L}_{1}$. Thus $\mathscr{P}^{-}(p)=f_{2}\left(f_{1}(p)\right)$.

considered curves of knees, since cell 2 is at a local max or min on each.)

To factor $\mathscr{P}^{+}$, we must keep track of both cell 1 and cell 2 . This time we factor the full map instead of just the cell 1 component. Here $g_{1}$ has domain in $\hat{L}_{1}$ and maps into $\hat{L}_{2}$; it is defined by computing the time $\tau_{1}(p)$ it takes for the point $j(p) \in H_{1}$ to reach $M_{1}$, and letting $g_{1}(p)$ be the point in $\hat{L}_{2}$ that flows from $j\left(M_{2}\right)$ for time $\tau_{1}(p)$ (see Fig. 15 and compare to Fig. 10). By assumption H4, $g_{1}(p)$ is below $m_{2}$. The function $g_{2}$ takes points in $\hat{L}_{2}$ into $\hat{L}_{1}$. For a point $q \in \hat{L}_{2}$, let $\tau_{2}(q)$ be the time from $j(q) \in H_{2}$ to flow to $M_{2}$, and let $g_{2}(q)$ be the point reached on $\hat{L}_{1}$ after flowing from $j\left(M_{1}\right) \in \hat{L}_{1}$ for time $\tau_{2}(q)$. (Note that both of the 


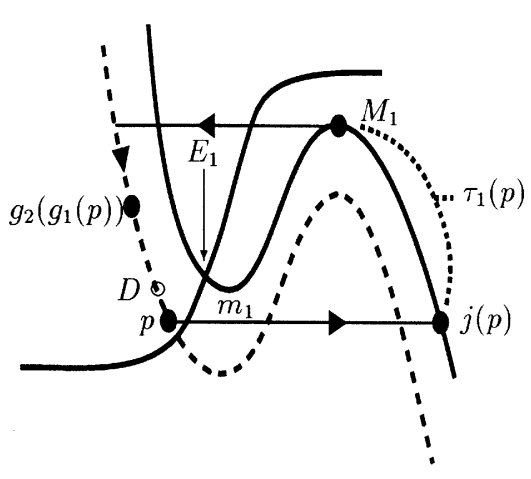

1

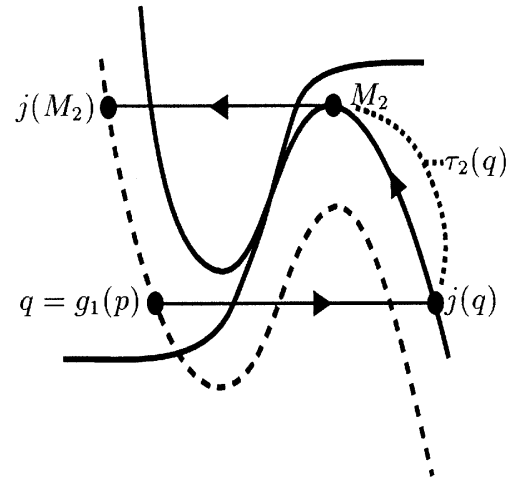

2

Fig. 15. The functions $g_{1}$ and $g_{2} . g_{1}$ maps $p \in \hat{L}_{1}$ to $g_{1}(p) \in \hat{L}_{2} . g_{2}$ maps $q \in \hat{L}_{2}$ to $g_{2}(q) \in \hat{L}_{1}$. Thus $\mathscr{P}^{+}(p)=g_{2}\left(g_{1}(p)\right)$.

above are maps from one curve of knees to another.) By construction, $g_{2}(q)=g_{2}\left(g_{1}(p)\right)=\mathscr{P}^{+}(p)$.

We now discuss conditions under which $\mathscr{P}$ is in case I or case II. In case II we have $\mathscr{P}^{-}(D)<\mathscr{P}^{+}(D)$ i.e.

$$
f_{2} \circ f_{1}(D)<g_{2} \circ g_{1}(D) .
$$

We will compute appropriate bounds on the left and right hand sides of this inequality to provide sufficient conditions for $\mathscr{P}$ being in case I or case II. For case II, this bound will be interpreted in terms of physiological properties of cell 1 and cell 2 . We also give additional conditions for the interesting case IIc.

Proposition 4.2. Let $q_{D}=g_{1}(D)$. If $\tau_{2}\left(q_{D}\right)-\tau_{2}\left(m_{2}\right)$ is greater than the time to flow from $j\left(M_{1}\right)$ to $D$ on $\hat{L}_{1}$ then $\mathscr{P}$ is in case I (see Fig. 15).

Proof. Let $E_{1}$ denote the critical point on branch $L_{1}$. Since $m_{1}<E_{1}$ and $D=j\left(m_{1}\right)$ we know that $m_{1}<f_{1}(D)$. This implies that $f_{2}\left(m_{1}\right)<f_{2} \circ f_{1}(D)$ and thus $f_{2}\left(m_{1}\right)$ is a lower bound of $f_{2}$. Denote the flow on $\hat{L}_{1}$ by $w_{1}=w_{L}\left(t ; w_{0}\right)$. Since $f_{2}\left(m_{1}\right)$ is the point found by flowing on $\hat{L}_{1}$ from $j\left(m_{1}\right)=D$ for time $\tau_{2}\left(m_{2}\right)$, we have

$$
f_{2}\left(m_{1}\right)=w_{L}\left(\tau_{2}\left(m_{2}\right) ; D\right) .
$$

By construction, $g_{2}\left(q_{D}\right)$ is the point on $\hat{L}_{1}$ determined by flowing from $j\left(M_{1}\right)$ for time $\tau_{2}\left(q_{D}\right)$ so that

$$
g_{2}\left(q_{D}\right)=w_{L}\left(\tau_{2}\left(q_{D}\right) ; j\left(M_{1}\right)\right)
$$

By the group property, flowing from $j\left(M_{1}\right)$ for time $\tau_{2}\left(q_{D}\right)$ is the same as first flowing from $j\left(M_{1}\right)$ for time $\tau_{2}\left(q_{D}\right)-\tau_{2}\left(m_{2}\right)$ and then flowing from 
this point for time $\tau_{2}\left(m_{2}\right)$. That is,

$$
\begin{aligned}
g_{2}\left(q_{D}\right) & =w_{L}\left(\tau_{2}\left(q_{D}\right) ; j\left(M_{1}\right)\right) \\
& =w_{L}\left(\tau_{2}\left(m_{2}\right) ; w_{L}\left(\tau_{2}\left(q_{D}\right)-\tau_{2}\left(m_{2}\right) ; j\left(M_{1}\right)\right)\right) .
\end{aligned}
$$

By assumption, $w_{L}\left(\tau_{2}\left(q_{D}\right)-\tau_{2}\left(m_{2}\right) ; j\left(M_{1}\right)\right)<D$. Since $f_{2}\left(m_{1}\right)$ is found by flowing from $D$ for time $\tau_{2}\left(m_{2}\right)$ and $g_{2}\left(q_{D}\right)$ is found by flowing from a point below $D$ also for time $\tau_{2}\left(m_{2}\right)$ it follows that

$$
g_{2} \circ g_{1}(D)=g_{2}\left(q_{D}\right)<f_{2}\left(m_{1}\right)<f_{2} \circ f_{1}(D) .
$$

Let $\hat{E}_{2}$ denote the critical point on branch $\hat{L}_{2}$.

Proposition 4.3. Let $\tau_{2}\left(m_{2}\right)$ denote the time to flow from $j\left(m_{2}\right)$ to $M_{2}$ on branch $\mathrm{H}_{2}$ and let $\tau_{2}\left(\hat{E}_{2}\right)$ denote the time to flow from $j\left(\hat{E}_{2}\right)$ to $M_{2}$ on $H_{2}$. If the time to flow from $j\left(M_{1}\right)$ to $j\left(E_{1}\right)$ on $\hat{L}_{1}$ is greater than $\tau_{2}\left(\hat{E}_{2}\right)-\tau_{2}\left(m_{2}\right)$ then (21) holds and $\mathscr{P}$ is in case II (see Fig. 16).

The physiological interpretation of Proposition 4.3 is that if the recovery time of cell 1 is greater than the additional time cell 2 fires due to inhibition then $\mathscr{P}$ is in case II (see Fig. 16).

Proof. Since $g_{1}: \hat{L}_{1} \mapsto \hat{L}_{2}$ and all trajectories on $\hat{L}_{2}$ are bounded below by the critical point $\hat{E}_{2}$, it follows that $\hat{E}_{2}<g_{1}(D)$. Since $g_{2}$ is orientation preserving,

$$
g_{2}\left(\hat{E}_{2}\right)<g_{2} \circ g_{1}(D)
$$

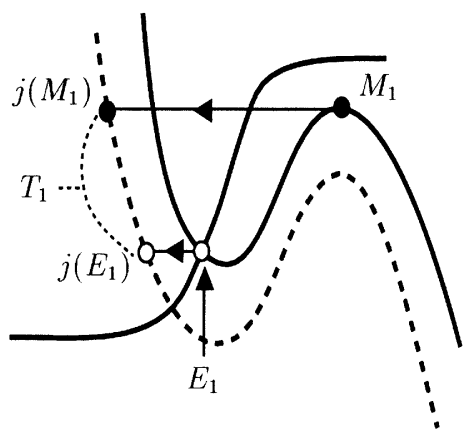

1

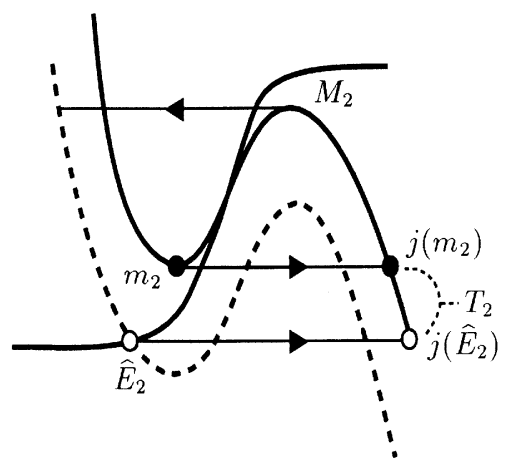

2

Fig. 16. The graphical interpretation of (28). If the time to flow from $j\left(M_{1}\right)$ to $j\left(E_{1}\right)$ on $\hat{L}_{1}$ (denoted $\left.T_{1}\right)$ is greater than $\tau_{2}\left(\hat{E}_{2}\right)-\tau_{2}\left(m_{2}\right)$ (denoted $\left.T_{2}\right)$ then $(28)$ holds and $\mathscr{P}$ is in case II. 
Similarly, since $m_{1}<E_{1}$ and $D=j\left(m_{1}\right)$ it follows that $f_{1}(D)<E_{1}$. Hence, since $f_{2}$ is orientation preserving,

$$
f_{2} \circ f_{1}(D)<f_{2}\left(E_{1}\right) .
$$

It now follows from (26) and (27) that if

$$
f_{2}\left(E_{1}\right)<g_{2}\left(\hat{E}_{2}\right)
$$

then $\mathscr{P}$ is in case II.

By construction, $g_{2}\left(\hat{E}_{2}\right)$ is the result of flowing from $j\left(M_{1}\right)$ on $\hat{L}_{1}$ for time $\tau_{2}\left(\hat{E}_{2}\right)$ and $f_{2}\left(E_{1}\right)$ is the result of flowing from $j\left(E_{1}\right)$ on $\hat{L}_{1}$ for time $\tau_{2}\left(m_{2}\right)$ (see Fig. 16). If the time to flow from $j\left(M_{1}\right)$ to $j\left(E_{1}\right)$ on $\hat{L}_{1}$ is greater than $\tau_{2}\left(\hat{E}_{2}\right)-\tau_{2}\left(m_{2}\right)$ then (19) holds and $\mathscr{P}$ is in case II.

We now compute $d \mathscr{P}^{ \pm} / d p$ starting with $\mathscr{P}^{-}$. We use the factorization $\mathscr{P}^{-}=f_{2} \circ f_{1}$. In time coordinates, the flow portion of $f_{1}$ on $\hat{L}_{1}$ or $f_{2}$ on $L_{1}$ is translation by a fixed amount of time determined by the cell 2 trajectory. Thus $d f_{i} / d p=d j_{i} / d p$, where $j_{i}$ is the jump associated with $f_{i}$. The derivatives of such jumps were computed in Sects. 3.3 and 3.4 in a more general context. In our case, they are

$$
\begin{aligned}
& d j_{1} / d p=C_{\hat{L}_{1} L_{1}}(p) \\
& d j_{2} / d q=C_{L_{1} \hat{L}_{1}}(q)=1 / C_{\hat{L}_{1} L_{1}}(j(q))
\end{aligned}
$$

where $p \in \hat{L}_{1}$ is the origin of jump $1, q=f_{1}(p) \in L_{1}$ is the origin of jump 2 and $j(q)$ is the point on $\hat{L}_{1}$ at the same height as $q$ (see Fig. 9). Thus,

$$
\frac{d \mathscr{P}^{-}}{d p}=\frac{C_{\hat{L}_{1} L_{1}}(p)}{C_{\hat{L}_{1} L_{1}}(j(q))} .
$$

We compute $d \mathscr{P}^{+} / d p$ using $\mathscr{P}=g_{2} \circ g_{1}$. Again using time coordinates, we have that $d g_{i} / d p=d j_{i} / d p$, where $j_{i}$ is the jump associated with $g_{i}$. Note that each jump $j_{i}$ is now from $\hat{L}_{i}$ to $H_{i}$. By the constructions in Sect. 3.3, we have that

$$
\frac{d \mathscr{P}^{+}}{d p}=C_{\hat{L}_{2} H_{2}}\left(g_{1}(p)\right) C_{\hat{L}_{1} H_{1}}(p) .
$$

It is easy to see that for graphs of the form in Fig. 13, the overlapping case cannot occur when each branch of $\mathscr{P}$ has slope less than one. The slopes are determined by (30) and (31), but it is not immediate how the functions in (20) determine the numbers in (30) and (31). We now relate the latter derivatives to the flows on the slow manifolds. 
We start with (30), which is the more subtle. For a jump from $\hat{L}_{1}$ to $L_{1}$, it follows from (19) in Sect. 3 that

$$
C_{\hat{L}_{1} L_{1}}(p)=\frac{G_{1}\left(\hat{v}_{1}\left(w_{1}\right), w_{1}\right)}{G_{1}\left(v_{1}\left(w_{1}\right), w_{1}\right)}
$$

where $w_{1}$ is the $w_{1}$-component of $p, v_{1}\left(w_{1}\right)$ and $\hat{v}_{1}\left(w_{1}\right)$ are the $v_{1}$-components along $L_{1}$ and $\hat{L}_{1}$ of $j(p)$ and $p$ respectively. A similar formula holds for the other factor in (30).

The following observations provide a simple criterion for showing that this ratio of compression numbers is less than one. We first note that $C_{\hat{L}_{1} L_{1}}(p)$ is undefined at $j\left(E_{1}\right)$ since the vector field on $L_{1}$ is 0 at $E_{1}$. If $p>j\left(E_{1}\right)$ then $p>j(q)>j\left(E_{1}\right)$. Thus it suffices to show that $C_{\hat{L}_{1} L_{1}}(p)$ is positive and increases as the point $p$ moves down the curve (i.e. that $d C_{\hat{L}_{1} L_{1}} / d p<0$ ) for points above $j\left(E_{1}\right)$. This implies that the denominator is larger than the numerator in (30). Since the direction of flow on $L_{1}$ and $\hat{L}_{1}$ is the same for points above $j\left(E_{1}\right)$, it follows from (32) that $C_{\hat{L}_{1} L_{1}}(p)>0$ in this region. On the other hand, if $p<j\left(E_{1}\right)$ then $p<j(q)<j\left(E_{1}\right)$. Thus for points below $j\left(E_{1}\right)$ we must show that $C_{L_{1} L_{1}}(p)$ is negative and $d C_{\hat{L}_{1} L_{1}} / d p<0$. In this region the flow is downward on $L_{1}$ and upward on $\hat{L}_{1}$ so that $C_{\hat{L}_{1} L_{1}}(p)<0$. We shall use (32) to determine when $d C_{\hat{L}_{1} L_{1}} d d p<0$ and hence when $d \mathscr{P}^{-} / d p<1$. We shorten the notation in (32) by writing $G_{1}\left(v_{1}\left(w_{1}\right), w_{1}\right)$ as $G_{1}\left(w_{1}\right)$ and $G_{1}\left(\hat{v}_{1}\left(w_{1}\right), w_{1}\right)$ as $\hat{G}_{1}(w)$. The conditions below are closely related to those introduced in [18] to get stable antiphase solutions between two oscillators coupled via excitatory fast threshold modulation.

Proposition 4.4. Suppose that

$$
\begin{array}{r}
\frac{d}{d w_{1}}\left[\widehat{G}_{1}\left(w_{1}\right)-G_{1}\left(w_{1}\right)\right]>0, \\
\frac{d}{d w_{1}} \hat{G}_{1}\left(w_{1}\right)<0, \\
{\left[G_{1}\left(w_{1}\right)-\hat{G}_{1}\left(w_{1}\right)\right]>0 .}
\end{array}
$$

Then $d C_{\hat{L}_{1} L_{1}} / d p<0$.

Remark 4.2. In [18], it was shown that families of equations standardly used to model voltage-gated conductance equations satisfy the hypotheses of Proposition 4.4. For the geometric interpretation of these conditions see [18]. 
Proof of Proposition 4.4. Using (32) and the quotient rule, $d C_{\hat{L}_{1} L_{1}} / d p$ has the same sign as

$$
\begin{aligned}
\hat{G}_{1}^{\prime}\left(w_{1}\right) G_{1}\left(w_{1}\right)-G_{1}^{\prime}\left(w_{1}\right) \hat{G}_{1}\left(w_{1}\right)= & \widehat{G}_{1}\left(w_{1}\right) \frac{d}{d w_{1}}\left[\hat{G}_{1}\left(w_{1}\right)-G_{1}\left(w_{1}\right)\right] \\
& +\hat{G}_{1}^{\prime}\left(w_{1}\right)\left[G_{1}\left(w_{1}\right)-\hat{G}_{1}\left(w_{1}\right)\right]
\end{aligned}
$$

In the first product, the first factor is negative by general assumption about the geometry and the second is positive by (33). In the second product, the first factor is negative by (34) and the second is positive by (35). Thus (36) is less than zero.

We now go to $d \mathscr{P}^{+} / d p$ and evaluate (31) more explicitly. It is easier in this case than in (30) to see when the expression is less than one, because each of the factors of (31) is less than one in absolute value for a large class of equations. The first factor of (31) may be written as

$$
\frac{G_{2}\left(\hat{v}_{2}\left(w_{2}\right), w_{2}\right)}{G_{2}\left(v_{2}\left(w_{2}\right), w_{2}\right)}
$$

where now the $v_{2}\left(w_{2}\right)$ and $\hat{v}_{2}\left(w_{2}\right)$ denote the values of $v_{2}$ along the curves $H_{2}$ and $\hat{L}_{2}$ respectively. Similarly, the second factor of (31) may be written as

$$
\frac{G_{1}\left(\hat{v}_{1}\left(w_{1}\right), w_{1}\right)}{G_{1}\left(v_{1}\left(w_{1}\right), w_{1}\right)} .
$$

That (37) and (38) be less than one in absolute value is the statement that there is compression across the jump between these branches. It was shown in [18] that such compressions are standard features of voltage-gated conductance equations used to model nerve cells. The basic idea behind this argument is that for $i=1,2, G_{i}\left(v_{i}, w_{i}\right)$ is "small" since $w_{i}$ is "close" to the critical point on $\hat{L}_{i}$ and $G_{i}\left(v_{i}, w_{i}\right)$ is "large" on $H_{i}$ since $w_{i}$ is "far" from the $w_{i}$-nullcline defined by $G\left(v_{i}, w_{i}\right)=0$. This is made explicit in [18]. The above results are summarized as follows.

Proposition 4.5. If equations (20) satisfy the hypotheses of Propositions 4.3 and 4.4 then the map $\mathscr{P}$ is in case IIC.

\section{Dynamics due to the slow rise time of another current}

In this section we return to the full five-dimensional equations. The addition of the new current allows the introduction of two new time scales, the rise time of the new current during the silent period, and the 
reset time of that current during the active period. We show that by making the rise time sufficiently slow (but not necessarily an order of magnitude slower than, e.g., the cell 1 recovery time), the system can again be reduced to a one-dimensional map. The behavior of this one-dimensional map depends critically on the reset time. The full equations have the form

$$
\begin{aligned}
d v_{1} / d \tau & =F_{1}\left(v_{1}, w_{1}, s ; I_{1}\right)+\bar{g}\left(v_{2}\right)\left(v_{s y n}-v_{1}\right) \\
d w_{1} / d \tau & =\varepsilon G_{1}\left(v_{1}, w_{1}\right) \\
d s / d \tau & =\varepsilon\left[s_{\infty}\left(v_{1}\right)-s\right] / \lambda\left(v_{1}\right) \\
d v_{2} / d \tau & =F_{2}\left(v_{2}, w_{2} ; I_{2}\right)+\bar{g}\left(v_{1}\right)\left(v_{s y n}-v_{2}\right) \\
w_{2} / d \tau & =\varepsilon G_{2}\left(v_{2}, w_{2}\right) .
\end{aligned}
$$

Details are given in the Appendix.

To get the reduction, we note that the fast equations for the fivedimensional equations have the form

$$
\begin{aligned}
& d v_{1} / d \tau=F_{1}\left(v_{1}, w_{1}, s ; I_{1}\right)+\bar{g}\left(v_{2}\right)\left(v_{1}-v_{s y n}\right) \\
& d v_{2} / d \tau=F_{2}\left(v_{2}, w_{2} ; I_{2}\right)+\bar{g}\left(v_{1}\right)\left(v_{2}-v_{s y n}\right)
\end{aligned}
$$

and the slow equations have the form

$$
\begin{aligned}
d w_{1} / d t & \left.=G_{1}\left(v_{1}, w_{1}\right)=\left[w_{\infty}\left(v_{1}\right)\right)-w_{1}\right] / \lambda_{w}\left(v_{1}\right) \\
d s / d t & =\left[s_{\infty}\left(v_{1}\right)-s\right] / \lambda_{s}\left(v_{1}\right) \\
d w_{2} / d t & \left.=G_{2}\left(v_{2}, w_{2}\right)=\left[w_{\infty}\left(v_{2}\right)\right)-w_{2}\right] / \lambda_{w}\left(v_{2}\right)
\end{aligned}
$$

where $v_{1}=v_{1}\left(w_{1}, s\right)$ and $v_{2}=v_{2}\left(w_{2}\right)$. Figure 17 shows the nullsurfaces $d v_{1} / d \tau=0$, with and without inhibition, and the nullsurface $d w_{1} / d \tau=0$. Each of these is two-dimensional in $\left(v_{1}, w_{1}, s\right)$ space, and are the analogues of the curves shown in Fig. 7 for cell 1. The analogue of the local maxima and minima $M_{1}, \hat{M}_{1}$, and $m_{1}, \hat{m}_{1}$ are now the curves $M_{1}(s), \hat{M}_{1}(s)$, and $m_{1}(s), \hat{m}_{1}(s)$. Cell 2 remains two-dimensional with nullclines as in Fig. 7.

The natural singular Poincare map associated with this new setup is a function from the knee of the inhibited LH branch to itself. In other words, cell 2 is at $M_{2}$ (as before) and cell 1 is on the inhibited low branch $\hat{L}_{1}$ which is now two-dimensional. Thus the domain of this singular Poincare map is two-dimensional. As before, the domain is divided into two parts (before by a point, this time by a curve) corresponding to whether or not cell 1 will fire in that cycle of cell 2. As before, the dividing curve is the image of the local minimum of $L_{1}$ (in 


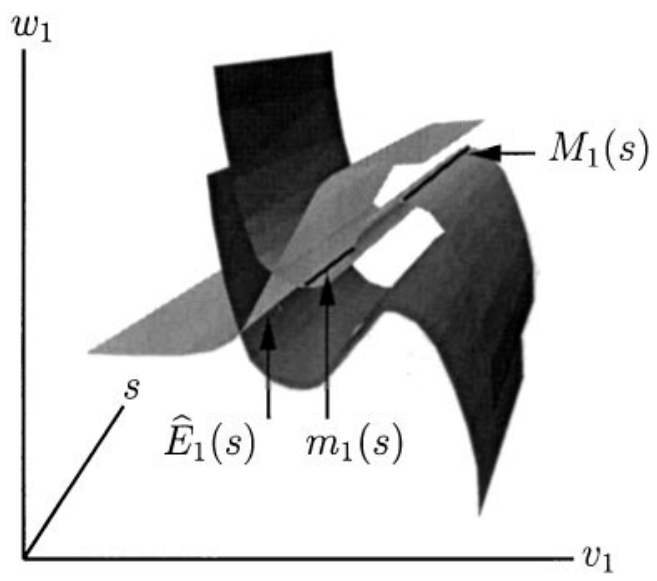

Fig. 17. The inhibited and uninhibited $v_{1}$-nullclines of cell 1 with $i_{h}$. Because of the addition of the variable $s$ describing $i_{h}$, the phase space associated with cell 1 is now three-dimensional and the corresponding nullclines are two-dimensional. The knees $M_{1}$ and $m_{1}$ are curves parameterized by $s$ as are the intersections of the $w_{1}$-nullcline with the inhibited and uninhibited $v_{1}$-nullclines.

this case $\left.m_{1}(s)\right)$ in $\hat{L}_{1}$ under the jump map. That curve is the analogue of the point $D$ in the previous section.

Nontrivial dynamics correspond to trajectories that spend some time in each of the two domains. In the previous section, such trajectories arose from the long time scale of the recovery variable of cell 1 , making it possible for more than one cycle of cell 2 to happen before cell 1 is ready to fire again. In this section, the mechanism giving rise to the interesting dynamics is fundamentally different, and depends more on the interplay of different time scales than before.

In this case, the recovery time of cell 1 is comparable to (or faster than) the burst time of cell 2 . This implies that at the end of a burst of cell 2, a cell 1 trajectory is near the intersection of the surface $\dot{w}_{1}=0$ and the surface $\hat{L}_{1}$. This intersection is a curve we shall call $\hat{E}_{1}(s)$, the analogue of the point $\hat{E}_{1}$ in Sect. 4 . If $s$ evolves very slowly (so can be treated as a parameter), a trajectory would fire or not in a given cycle depending only on whether the point on $\hat{E}_{1}(s)$ is above or below the point on $j\left(m_{1}(s)\right)$ with the same value of $s$ (see Fig. 18). If the curves $\hat{E}_{1}(s)$ and $j\left(m_{1}(s)\right)$ cross, then nontrivial dynamics can occur when trajectories go back and forth across this intersection. Note that $\hat{E}_{1}(s)$ plays a more central role in this scenario than $\hat{E}_{1}$ does in Sect. 4.

This scenario does not require $s$ to evolve an order of magnitude more slowly than the recovery variable of cell 1 on $\hat{L}_{1}$, but if it does not, one must be a little more careful in the description. With a milder hypothesis, it is still true that the system can be approximated by 


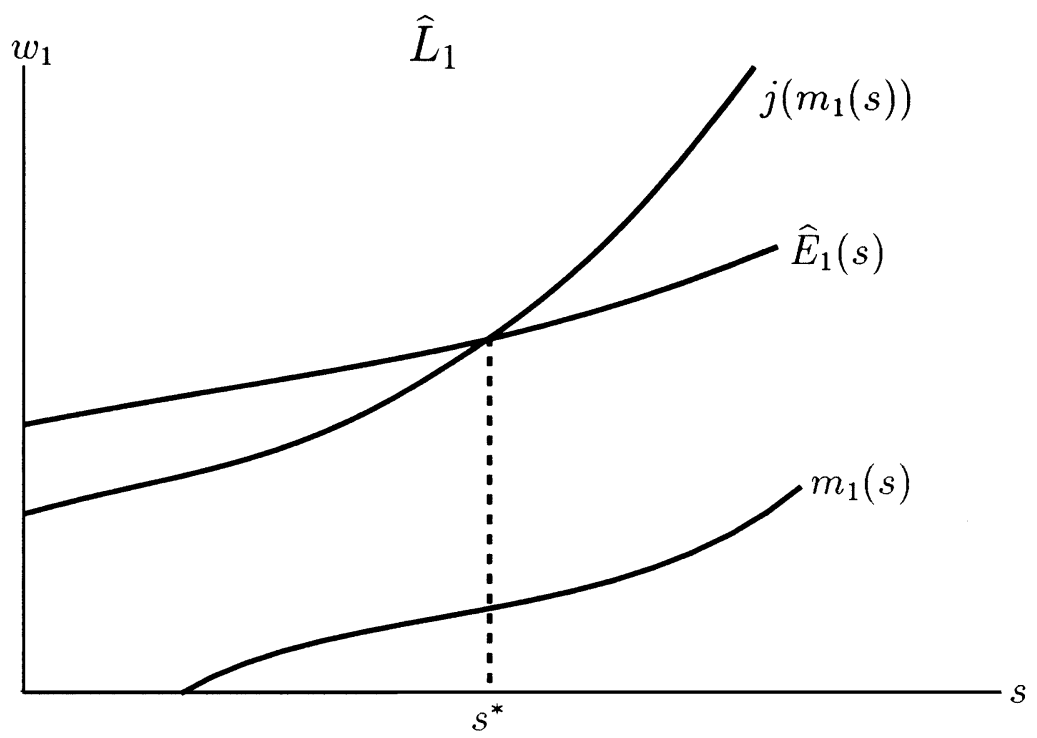

Fig. 18. The projection of $\hat{L}_{1}$ onto the $\left(s, w_{1}\right)$ plane. To the left of $s^{*}, \hat{E}_{1}(s)$ is above $j\left(m_{1}(s)\right)$ and hence trajectories jumping from this segment of $\hat{E}_{1}(s)$ jump to the uninhibited lower branch $L_{1}$. To the right of $s^{*}, \hat{E}_{1}(s)$ is below $j\left(m_{1}(s)\right)$ and trajectories jumping from here jump to the uninhibited high branch $H_{1}$.

a Poincaré map whose domain is one-dimensional rather than two. However, the curve of this domain is not necessarily $\hat{E}_{1}(s)$ but a curve that approaches it as the rate of change of $s$ decreases to zero. More specifically, consider the first two equations of (41), with $v=v_{1}\left(w_{1}, s\right)$ and $v_{2}=v_{2}\left(w_{2}\right)$ chosen to correspond to dynamics of $\hat{L}_{1}$ (i.e., cell 2 firing). Under hypothesis (H2) given below, these equations have a critical point at the intersection of $s=1$ and $\hat{E}_{1}(s)$. Further hypotheses ( $\mathrm{H} 4$ and H5) imply that the eigenvalues of the linearization at this critical point are real, negative, and unequal. We shall refer to any trajectory tangent to the eigenvector of the least negative eigenvalue as a "slow stable manifold (SSM)." All solutions on $\hat{L}_{1}$ having an initial condition with $s$ 丰 1 approach the critical point tangent to SSM. The smaller the ratio $\neq \lambda_{w}\left(v_{1}\right) / \lambda_{s}\left(v_{1}\right)$ on $\hat{L}_{1}$ in (41) the faster solutions approach SSM. Moreover, as this ratio goes to zero, SSM approaches the curve $\hat{E}_{1}(s)$. We use the curve $S S M$ as the domain of the reduced Poincare map; the actual Poincaré map, restricted to SSM, does not return exactly to $S S M$, but close to it and the image is then projected onto SSM.

In Sect. 5.1 we spell out the hypotheses under which the geometric picture described above is valid. In Sect. 5.2 we analyze different 
behaviors compatible with those hypotheses. These include the staircase ( $n: m$ coordination) phenomena of Sect. 4 , but also $n: 1$ coordination. We also comment here on how the mode of coordination ( $n: 1$ or $n: m)$ affects the regularity of the timing of the burst of the slower cell. In Sect. 5.3 we look at how parameters in the biophysical description of the equations affect the behavior of the reduced map.

\subsection{Hypotheses, slow manifolds and Poincare maps}

The equations for cell 2 are as in Sect. 4, and we assume the same hypotheses:

H1: Without inhibition, cell 2 is an oscillator. In the presence of inhibition it has a stable critical point. Its recovery time from bursting is fast enough compared with the firing time of cell 1 (the minimal time cell 1 spends on $H_{1}$ after a firing due to being released from inhibition) that cell 2 can fire on release of inhibition from cell 1 . This is similar to H4 of Sect. 4.1.

H2: For each fixed value of $s$, cell 1 has a stable critical point both without inhibition and in the presence of inhibition. On $\hat{L}_{1}$ this is the curve $\hat{E}_{1}(s)$. In addition, we assume that $s_{\infty}\left(v_{1}\right) \equiv 1$ on $L_{1}$ and $\hat{L}_{1}$ while $s_{\infty}\left(v_{1}\right) \equiv 0$ on $H_{1}$. Thus $s$ increases on $L_{1}$ and $\hat{L}_{1}$ and decreases on $H_{1}$.

Since $i_{h}$ is an inward cation current, the nullcline surface $d v_{1} / d \tau=0$ rises with increasing $s$. Thus we assume

H3: $m_{1}^{\prime}(s)>0, M_{1}^{\prime}(s)>0, \hat{E}_{1}^{\prime}(s)>0$. This hypothesis can be verified by direct calculation for the equations in the Appendix.

We now add timescale hypotheses. In Sect. 4, the central hypothesis that insures that cell 1 neither fires each cycle nor remains silent is that the recovery time of cell 1 is sufficiently long compared with the firing time of cell 2 . We shall assume the opposite here, that cell 1 recovers by the end of the firing time of cell 2:

H4: On $\hat{L}_{1}, \lambda_{w}\left(v_{1}\right)$ is sufficiently small compared to the firing time of cell $2\left(\lambda_{w}\left(v_{1}\right)\right.$ controls the recovery rate $d w_{1} / d t$ of cell 1$)$.

H5: On $L_{1}$ and $\hat{L}_{1}, \lambda_{s}\left(v_{1}\right)$ is independent of $v_{1}$ and sufficiently large relative to the firing time of cell 2 . We will denote the value by $\lambda_{L}$. Similarly, $\lambda_{s}\left(v_{1}\right)$ is independent of $v_{1}$ on $H_{1}$ and denoted $\lambda_{H}$.

To get nontrivial dynamics, we introduce another hypothesis that insures that cell 1 will fire only from a proper subset of $\hat{L}_{1}$. This hypothesis says that above some level of $i_{h}$ (i.e., value of $s$ ), cell 1 can 
rebound at the end of a cycle of inhibition from cell 2; below that level there is no inhibitory rebound.

H6: Let $j\left(m_{1}(s)\right)$ denote the image in $\hat{L}_{1}$ of the curve $m_{1}(s) \in L_{1}$. The curves $j\left(m_{1}(s)\right)$ and $S S M$ intersect in a unique point $s^{*}$, with $j\left(m_{1}(s)\right)$ below $S S M$ for $s<s^{*}$ and $j\left(m_{1}(s)\right)$ above $S S M$ for $s>s^{*}$. See Fig. 18.

As in Sect. 4, only three of the four branches of the slow manifold are relevant to periodic solutions, since no trajectory jumps to the $H H$ branch. Again, a trajectory leaves a branch when it hits a "manifold of knees," and these jump-off sets are cross-products of slow manifolds of cell 1 and cell 2, with one component at a local maximum or minimum. The statement of Proposition 4.1, about the destination of each jump, holds for the current situation as well. The proof is identical, with part (3) now a consequence of Hypotheses $\mathrm{H} 4$ and H6. As before, the singular Poincaré map is constructed by starting with cell 2 at $M_{2}$ and cell 1 at a point on $\hat{L}_{1} . \mathscr{P}$ is defined to be the resulting point on $\hat{L}_{1}$ when cell 2 next reaches $M_{2}$. $\mathscr{P}$ is again discontinuous, with a curve of discontinuities at $j\left(m_{1}(s)\right)$. We continue to use the notation $\mathscr{P}^{-}$(respectively $\mathscr{P}^{+}$) for the branch of $\mathscr{P}$ in which cell 1 does not fire (respectively fires).

From Hypotheses H4, and H5, we can define a "reduced singular Poincare map," one that is one-dimensional. We let $\mathscr{2}^{+}$(respectively $\mathscr{Q}^{-}$) be $\mathscr{P}^{+}$(respectively $\mathscr{P}^{-}$) restricted to $S S M$. In other words, given a $p \in S S M, \mathscr{2}(p)=\pi \circ \mathscr{P}(p)$ where $\pi$ is the projection from a tubular neighborhood $U$ of $S S M$ to $S S M$ that leaves the $s$-coordinate of $\mathscr{P}$ unchanged. Note that $\mathscr{Q}^{-}$is defined for $s \leqq s^{*}$ and $\mathscr{Q}^{+}$for $s \geqq s^{*}$. For $s=s^{*}$ the map $\mathscr{Q}$ is doubly defined by each of the one-sided limits.

\subsection{Different slopes, different behavior}

We shall now show that $\mathscr{2}^{-}$is always orientation preserving, but $2^{+}$could be either orientation preserving or reversing, depending on another time scale not yet mentioned. We shall then discuss the behavioral consequences of having the slope be negative.

For $\mathscr{Q}^{-}$, the argument is the same as for $\mathscr{P}^{-}$in Sect. 4 , this time using a parameterization in $s$ instead of $w_{1}$. As before, each jump and each flow is order preserving. This follows from (H2) and (H5) describing the properties of $d s / d t$ on $L_{1}$ and $\hat{L}_{1}$.

Now consider $\mathscr{2}^{+}$and let $p_{\alpha}, p_{\beta}$ be two points in the domain of $\mathscr{2}^{+}$, with the $s$-coordinate of $p_{\alpha}$ greater than the s-coordinate of $p_{\beta}$. After the first jump, the image of the points $p_{\alpha}$ and $p_{\beta}$ are in $H_{1}$, with unchanged values of $s$ and $w_{1}$. Thus, we continue to represent them on the same 


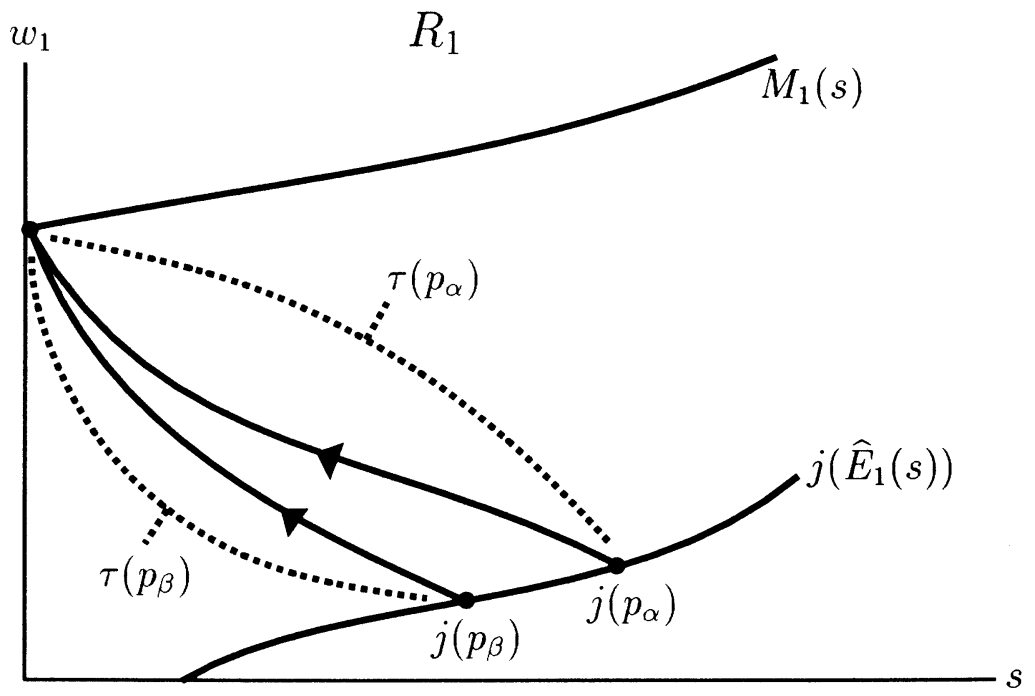

Fig. 19. Trajectories of $j\left(p_{\alpha}\right)$ and $j\left(p_{\beta}\right)$ under $(\mathrm{H} 7)$. Hypothesis $(\mathrm{H} 7)$ assumes that both trajectories intersect $M_{1}(s)$ near $s=0$. Because $j\left(\widehat{E}_{1}(s)\right)$ is increasing $\tau_{1}\left(p_{\alpha}\right)<\tau_{1}\left(p_{\beta}\right)$ under the conditions presented in (H8).

$\left(s, w_{1}\right)$ plane. The upper curve in Fig. 19 is $M_{1}(s)$. The points $j\left(p_{\alpha}\right), j\left(p_{\beta}\right)$ give rise to trajectories of the slow flow on $H_{1}$, which reach $M_{1}$ at times $\tau_{1}\left(p_{\alpha}\right), \tau_{1}\left(p_{\beta}\right)$ respectively. During this time, $s$ decreases and $w_{1}$ increases. The behavior of the system depends crucially on the time scale of $s$ during this flow.

Suppose, for example, that

H7: $s$ decreases fast enough along $H_{1}$ that $s \approx 0$ at the time a trajectory reaches $M_{1}(s)$.

Note that $\mathrm{H} 7$ does not require that $s$ decrease on the fast time scale of $v_{1}$, or even that it be much faster than $w_{1}$ on $H_{1}$. This is similar to hypothesis $\mathrm{H} 4$ which requires that $s$ increase slowly enough, (but not infinitely slow) on $\hat{L}_{1}$. Fig. 19 shows the slow trajectories of $j\left(p_{\alpha}\right)$ and $j\left(p_{\beta}\right)$ on $H_{1}$ under hypothesis H7. Suppose further that

H8: the $s$-coordinate of $p_{\alpha}$ greater than the $s$-coordinate of $p_{\beta}$ implies that $\tau_{1}\left(p_{\alpha}\right)<\tau_{1}\left(p_{\beta}\right)$.

This would be true, for example, if $d w / d t$ is dependent essentially on $w_{1}$ only, not on $v_{1}$, which can be affected by the value of $s$. Using $\mathrm{H} 3$ we see that such independence holds if $w_{\infty}(v)$ is almost independent of $v_{1}$ along $H_{1}$. $\mathrm{H} 8$ also holds if $s$ decreases on $H_{1}$ much faster than $w_{1}$ increases, so $s \approx 0$ for most of the trajectory. H8 can fail to hold if $d w_{1} / d t$ restricted to $H_{1}$ does depend in an essential way on $s$. 
Under hypotheses $\mathrm{H} 7$ and $\mathrm{H} 8,2^{+}$may be orientation reversing. To see this, we consider the second half of $\mathscr{Q}^{+}$, with a jump back to $\hat{L}_{1}$ and a flow along $\hat{L}_{1}$. Let $q_{\alpha}$ and $q_{\beta}$ be the points in $j\left(M_{1}\right) \times \hat{L}_{2}$ that are the images of the points $p_{\alpha}$ and $p_{\beta}$ in $\hat{L}_{1} \times M_{2}$ under the first half of $\mathscr{Q}^{+}$. H7 implies that the singular solutions originating at the cell 1 component of $q_{\alpha}, q_{\beta}$ both jump into a small neighborhood of the point having $s$-coordinate 0 and $w_{1}$-coordinate $m_{1}(0)$ on $\hat{L}_{1}$. After jumping, each solution flows until cell 2 reaches $M_{2}$. The times of this flow $\left(\tau_{2}\left(q_{\alpha}\right)\right.$ and $\left.\tau_{2}\left(q_{\beta}\right)\right)$ depend on the position of cell 2 on $\hat{L}_{2}$ when cell 1 jumps downward. $\mathrm{H} 8$ implies that the $w_{2}$-component for cell 2 is higher for the $\alpha$-trajectory. In other words, $\tau_{2}\left(q_{\alpha}\right)<\tau_{2}\left(q_{\beta}\right)$ (see Fig. 20). If this difference is large relative to the nearness of the $\alpha$ and $\beta$ solutions after the jump to $\hat{L}_{1}$ then $\mathscr{Q}^{+}\left(p_{\beta}\right)>\mathscr{2}^{+}\left(p_{\alpha}\right)$, so $\mathscr{2}^{+}$reverses orientation (see Fig. 21). Figure 24 shows a numerical approximation of the map $\mathscr{2}$ in this regime. Conditions guaranteeing orientation reversal are discussed in Sect. 5.3. Figure 24 shows a numerical approximation of the the graph of 2 in this parameter regime.

Now suppose we allow $s$ to decrease slowly enough on $H_{1}$ so that $\mathrm{H} 7$ is violated (see Fig. 22 and compare with Fig. 19). The flow on $\hat{L}_{1}$ after the down-jump is as in Fig. 23. If $\mathrm{H} 8$ is also violated so $\tau_{1}\left(p_{\alpha}\right)>\tau_{1}\left(p_{\beta}\right)$ then $\tau_{2}\left(q_{\alpha}\right)>\tau_{2}\left(q_{\beta}\right)$ and so $\mathscr{2}^{+}\left(p_{\alpha}\right)>\mathscr{2}^{+}\left(p_{\beta}\right)$, i.e. $\mathscr{2}^{+}$is orientation preserving. Figure 25 shows a numerical approximation of the map 2 in this regime. If $\mathrm{H} 7$ is violated but $\mathrm{H} 8$ holds, there may or may not be orientation reversal. Though we still have $\tau_{2}\left(q_{\alpha}\right)>\tau_{2}\left(q_{\beta}\right)$,

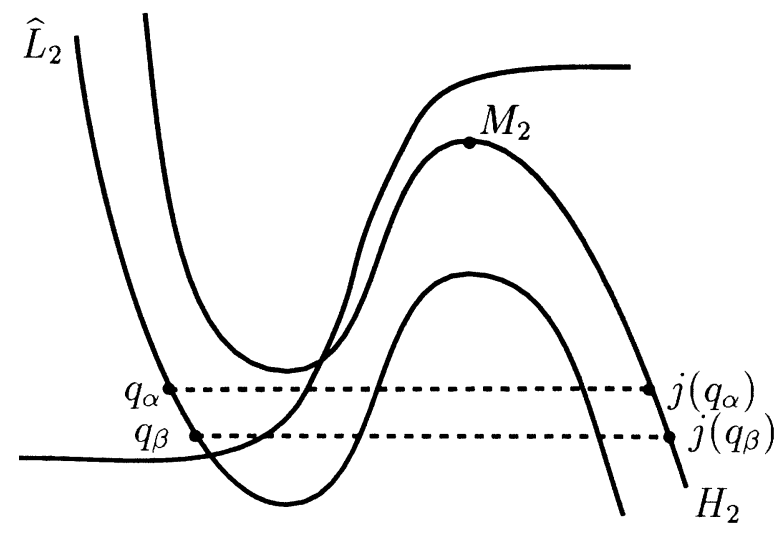

2

Fig. 20. The cell 2 portion of $2^{+}$. From (H7) and (H8), $\tau_{1}\left(p_{\alpha}\right)<\tau_{1}\left(p_{\beta}\right)$ implies that when the $\alpha$ and $\beta$ trajectories on $H_{1}$ reach the knee $M_{1}(s)$ the cell 2 component of $q_{\alpha}$ on $\hat{L}_{2}$ is above the cell 2 component of $q_{\beta}$ on the same branch. The jump to $H_{2}$ does not change this orientation. Since $\tau_{2}$ is the time to flow to $M_{2}$ it follows that $\tau_{2}\left(q_{\alpha}\right)<\tau_{2}\left(q_{\beta}\right)$. 


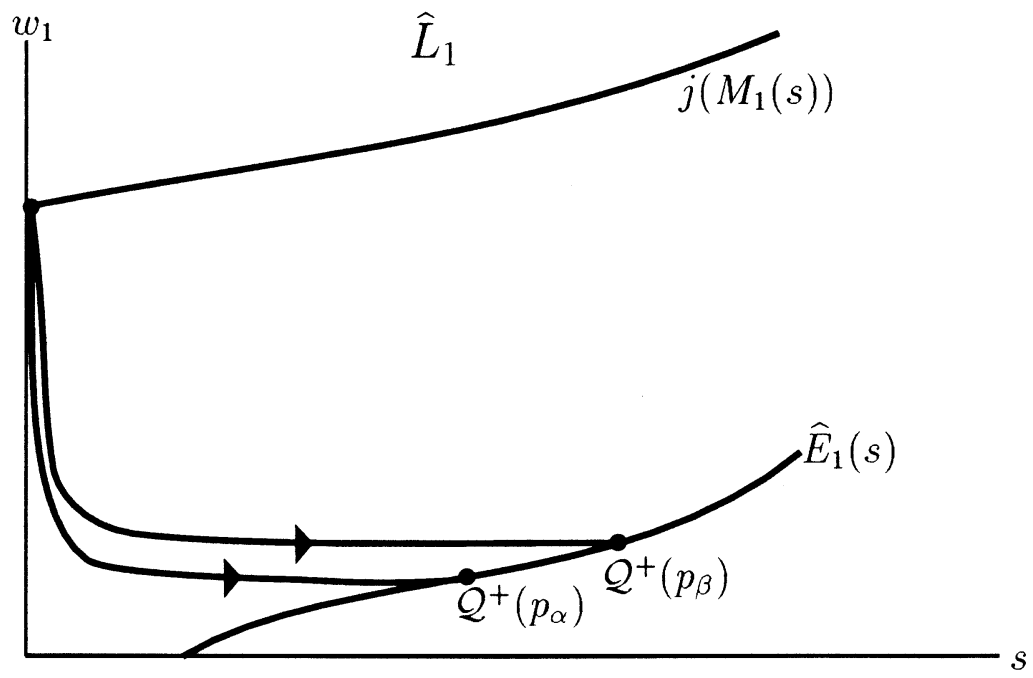

Fig. 21. The flow on $\hat{L}_{1}$ of $\mathscr{Q}^{+}$. Both the $\alpha$ and $\beta$ components on $\hat{L}_{1}$ start near $M_{1}(0)$. By (H6) only the value of the $s$-coordinate is dependent on the time of flow. If $\tau_{2}\left(q_{\alpha}\right)<\tau_{2}\left(q_{\beta}\right)$, it follows that $\mathscr{2}^{+}\left(p_{\alpha}\right)<\mathscr{2}^{+}\left(p_{\beta}\right)$ and hence $\mathscr{2}^{+}$is orientation reversing.

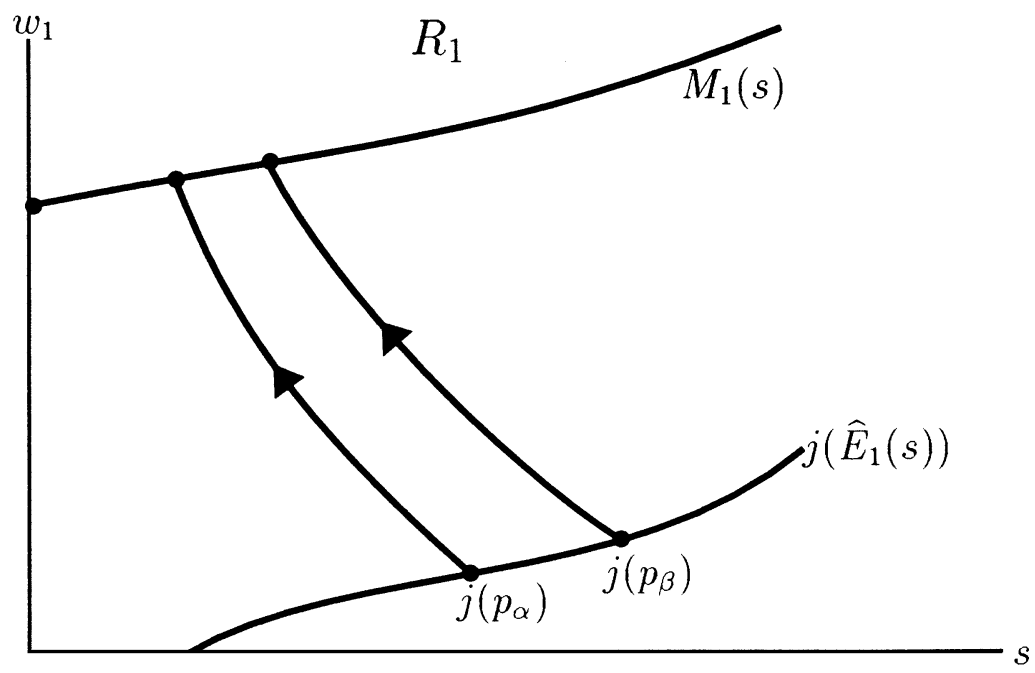

Fig. 22. Trajectories of $j\left(p_{\alpha}\right)$ and $j\left(p_{\beta}\right)$ when $(\mathrm{H} 7)$ is violated. When $(\mathrm{H} 7)$ is violated then the slow trajectories of $j\left(p_{\alpha}\right)$ and $j\left(p_{\beta}\right)$ are not close to $M_{1}(0)$ when reaching the knee.

the $\alpha$ and $\beta$ trajectories do not start sufficiently near each other (see Fig. 23) and the $\beta$-trajectory may or may not overtake the $\alpha$ trajectory in the longer time that it has to flow.

We have now seen that under hypotheses $\mathrm{H} 1-\mathrm{H} 8$, the resulting reduced singular Poincaré map may have $\left(\mathscr{Q}^{+}\right)^{\prime}(p)<0$. In Sect. 5.3, we 


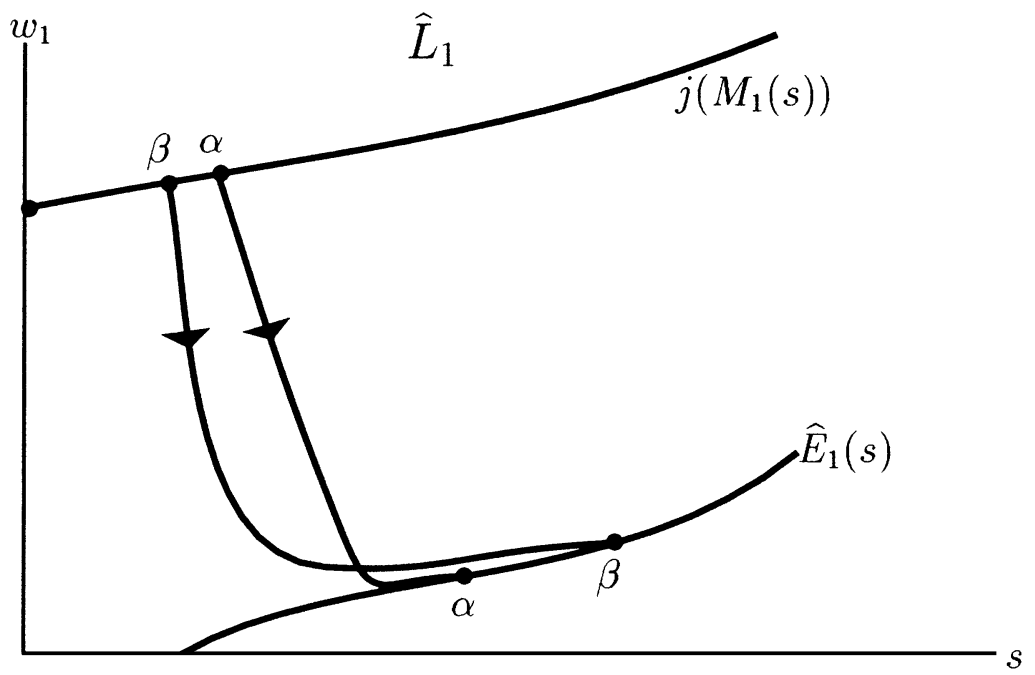

Fig. 23. An example of an orientation preserving $Q^{+}$when $(\mathrm{H} 7)$ is violated. If $(\mathrm{H} 8)$ holds (i.e. $\tau_{2}\left(q_{\alpha}\right)<\tau_{2}\left(q_{\beta}\right)$ ) it is still possible $Q^{+}\left(p_{\beta}\right)>Q^{+}\left(p_{\alpha}\right)$ since the $\alpha$ and $\beta$ components do not start from the same point on $j\left(M_{1}(s)\right)$.

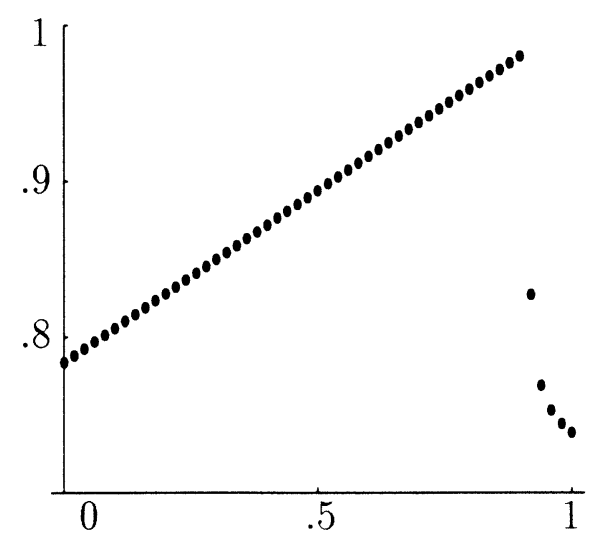

Fig. 24. A numerical approximation of the map $\mathscr{2}$ when $\mathscr{2}^{+}$is decreasing. This figure was generated by taking initial conditions near $\hat{E}_{1}(s)$ for cell 1 and near the point $M_{2}$ for cell 2. The initial condition for $s$ was incremented from 0 to 1 . For each $s, 2(s)$ was approximated by the value of $s$ when the $w_{2}$ coordinate of the trajectory reached a maximum.

will compute the slopes and provide conditions under which this condition on the derivative holds. In addition, we will show that if $p$ is given in time coordinates then $\left(\mathscr{Q}^{-}\right)^{\prime}(p)=1$ and $\left|\left(\mathscr{Q}^{+}\right)^{\prime}(p)\right|<1$ in parameter ranges related to the above discussion. We shall assume these properties in the description of behavior we give in the remainder of this section. 


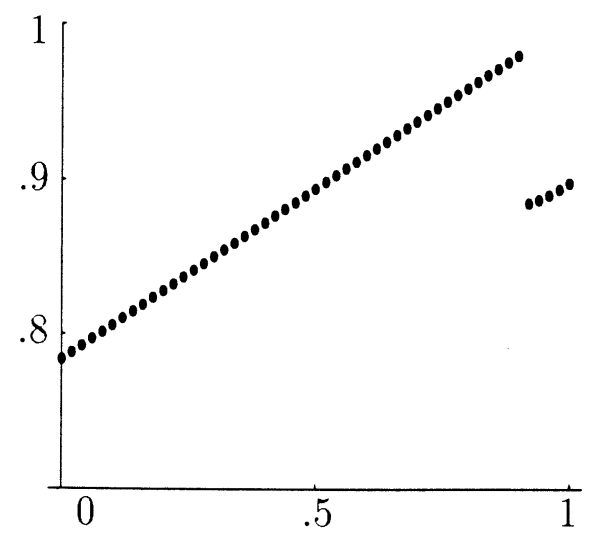

Fig. 25. A numerical approximation of the map 2 when $\mathscr{2}^{+}$is increasing. This figure was generated by taking initial conditions near $\hat{E}_{1}(s)$ for cell 1 and near the point $M_{2}$ for cell 2. The initial condition for $s$ was incremented from 0 to 1 . For each $s, 2(s)$ was approximated by the value of $s$ when the $w_{2}$ coordinate of the trajectory reached a maximum.

Figure 26 illustrates the two possible forms of the graph of $\mathscr{2}$ under the derivative restrictions imposed above. In A, $\mathscr{Q}^{-}\left(s^{*}\right)<\mathscr{2}^{+}\left(s^{*}\right)$ and $2^{+}$has an asymptotically stable fixed point. All points tend to this fixed point under iteration corresponding to $1: 1$ firing of cell 1 and cell 2. In $\mathrm{B}, \mathscr{2}^{-}\left(s^{*}\right)>\mathscr{Q}^{+}\left(s^{*}\right)$ and the interval $\left[\mathscr{Q}^{+}\left(\mathscr{Q}^{-}\left(s^{*}\right)\right), \mathscr{Q}^{-}\left(s^{*}\right)\right]$ is forward invariant under iteration of $\mathscr{2}$ and all points in the domain of $\mathscr{Q}$ are mapped into this interval. Thus it is sufficient to restrict our attention to this interval. The map $\mathscr{Q}$ is nonoverlapping (i.e. the graph of $2^{+}$is completely below the graph of $\mathscr{2}^{-}$) if and only if

$$
\mathscr{2}^{-}\left(\mathscr{Q}^{+}\left(\mathscr{Q}^{-}\left(s^{*}\right)\right)\right)>\mathscr{Q}^{+}\left(s^{*}\right)
$$

(see Fig. 26C). The derivative restrictions given above insure that (42) holds. If these conditions are relaxed then 2 may be overlapping and display chaotic behavior [23]. Maps having the qualitative features illustrated in Fig. 26B were analyzed in [23] and shown to have very different behavior from the maps discussed in Sect. 4. In particular, when $\mathscr{Q}$ is nonoverlapping the stable periodic solutions exhibited by these maps are associated only with stable $n: 1$ behavior of the two cells, with possible bistability of $n: 1$ and $(n+1): 1$ as some parameter (such as amount of hyperpolarizing current) is varied. A typical bifurcation diagram is shown in Fig. 27. The black and gray lines indicate distinct attracting periodic orbits. For parameter values having a single attracting orbit, only black is shown. For example, at $\alpha=0.2$ there exists an attracting fixed point (black) and an attracting period 2 orbit 


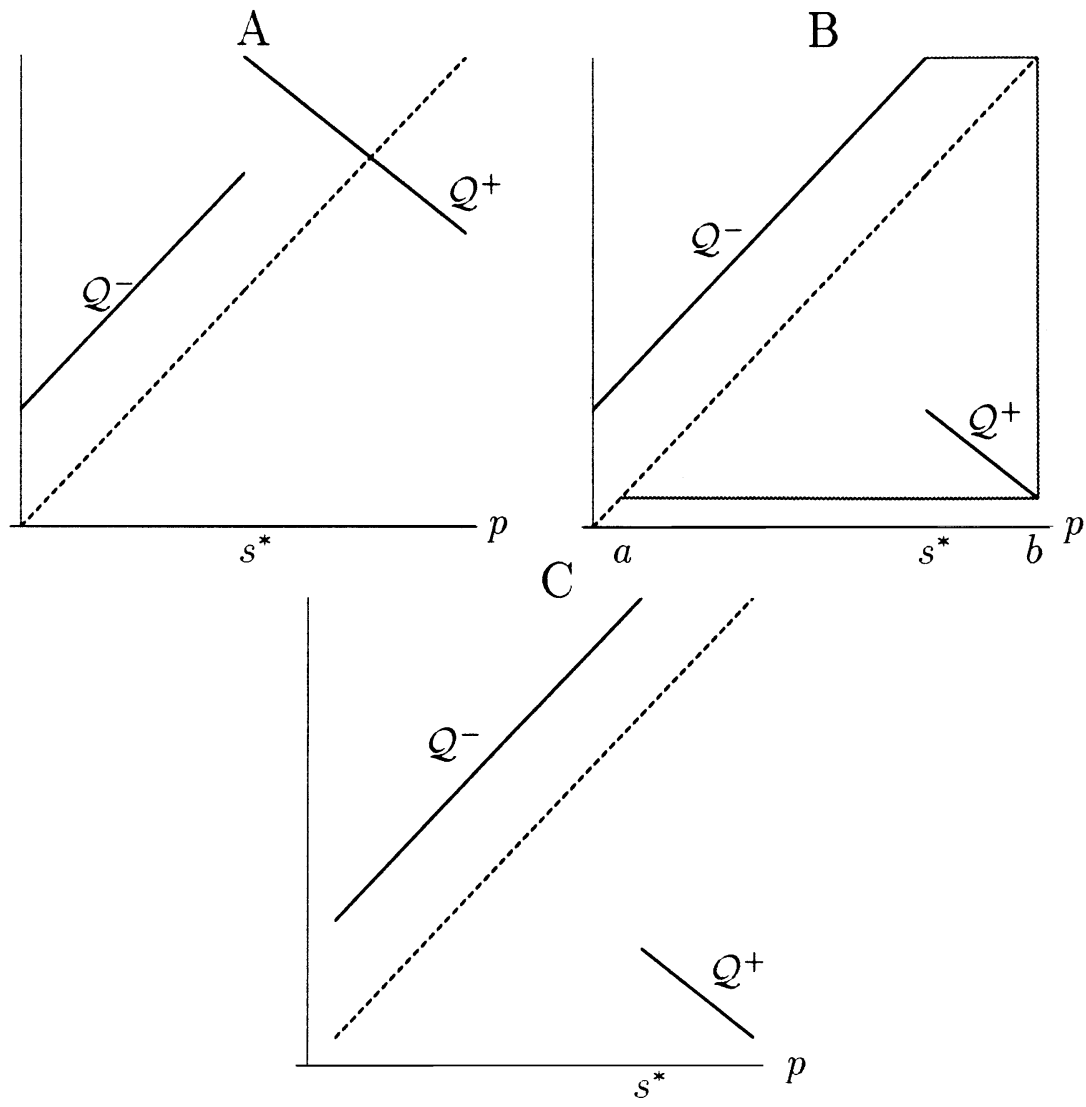

Fig. 26. Possible configurations of the graph of $\mathscr{2}$. In $\mathbf{A} \mathscr{2}^{-}\left(s^{*}\right)<\mathscr{Q}^{+}\left(s^{*}\right)$ and there exists an attracting fixed point for $2^{+}$. All points tend to this fixed point under iteration. This corresponds to 1: 1 firing of cell 1 and cell 2 . In $\mathbf{B} \mathscr{Q}^{-}\left(s^{*}\right)>\mathscr{Q}^{+}\left(s^{*}\right)$ and the interval $\left[\mathscr{2}^{+}\left(\mathscr{Q}^{-}\left(s^{*}\right)\right), \mathscr{2}^{-}\left(s^{*}\right)\right]$. is forward invariant. This interval is labeled $[a, b]$. In $\mathbf{C}$ we restrict to the invariant interval $\left[\mathscr{Q}^{+}\left(\mathscr{Q}^{-}\left(s^{*}\right)\right), \mathscr{Q}^{-}\left(s^{*}\right)\right]$. to show that (42) holds. In this scenario there exists at least one attracting periodic orbit of period $n$ and possibly a second attracting periodic orbit of period $n+1$. This corresponds to $n: 1$ and $(n+1): 1$ firing between cell 1 and cell 2 .

(gray) but at $\alpha=0.4$ there exists only an attracting period 2 orbit (black). The bifurcation between these these two types of behaviors occurs at approximately $\alpha=0.25$.

The nonoverlapping regime corresponds to the behavior seen in the motivating example given in Sect. 2. The bistability of period $n$ and $n+1$ orbits seen in Fig. 27 were also seen in the simulations of the model discussed in Sect. 2.

Remark 5.1. We can now see why maps with only $n: 1$ coordination have the property that the burst time of the slow cell remains the same from 


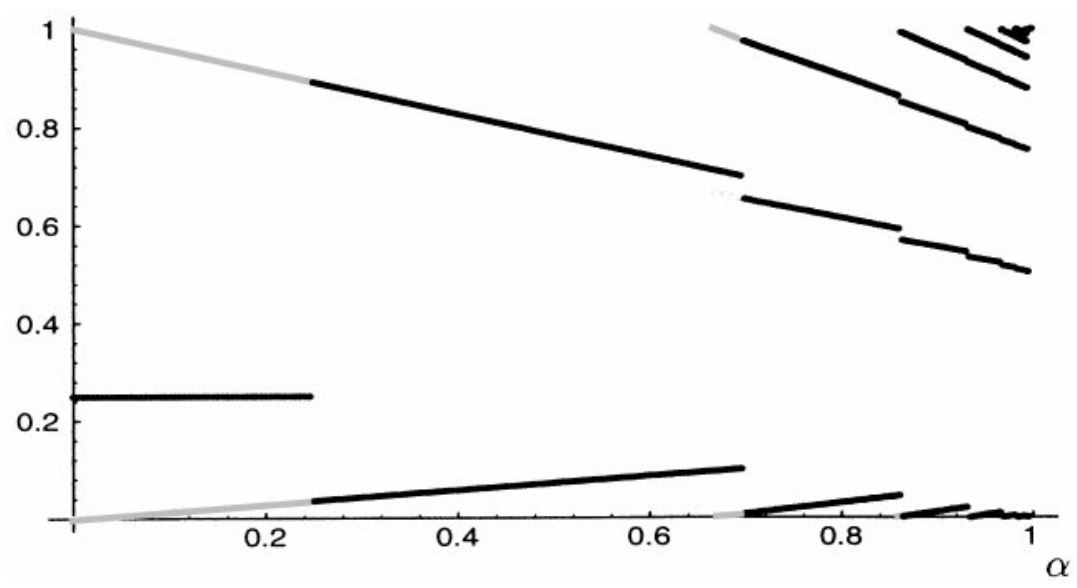

Fig. 27. A typical bifurcation diagram for a family of functions $\mathscr{Z}_{\alpha}$ satisfying the nonoverlapping condition. The black and gray lines indicate attracting periodic orbits. For example, at $\alpha=0.2$ there exists an attracting fixed point (black) and an attracting period 2 orbit (gray) but at $\alpha=0.4$ there exists only an attracting period 2 orbit (black).

burst to burst, while the maps with $n: m$ coordination need not display this kind of regulation. In the maps that display $n: m$ coordination, the number of bursts of the fast cell between bursts of the slow cell varies from cycle to cycle. This corresponds to variation (from slow cycle to slow cycle) in the position of the trajectory on $\hat{L}_{1}$ as it jumps to $H_{1}$. If the trajectory jumps from a lower value of $w_{1}$, the time to reach $M_{1}$ is longer and this time is (by definition) the burst time of the slow cell. By contrast, in the case of maps with n:1 coordination, the trajectory is strictly periodic, with the same number of fast cycles per slow cycle. For each slow cycle the trajectory then always jumps from the same point in the jump-up curve and hence has a constant burst time. See the discussion in Sect. 6 for further remarks.

\subsection{Biophysical parameters and slopes of $\mathscr{2}^{ \pm}$}

We now undertake a more rigorous look at the reduced map 2 to identify the essential biophysical factors that determine each of the scenarios discussed in Sect. 5.2. In particular, we will address the following questions.

1. What is $\left(\mathscr{Q}^{-}\right)^{\prime}(p)$ ?

2. When is $\mathscr{Q}^{+}\left(s^{*}\right)<\mathscr{2}^{-}\left(s^{*}\right)$ ?

3. Under what conditions does H8 hold true; i.e. when is $\tau_{1}(p)$ a decreasing function of the $s$-coordinate of $p$ ? 
4. If $\mathrm{H} 8$ holds, what additional properties are necessary to insure that $\left(\mathscr{Q}^{+}\right)^{\prime}(p)<0$ ?

5. When $\mathrm{H} 7$ and $\mathrm{H} 8$ hold true, under what conditions is 2 restricted to $\operatorname{Im}(2)$ nonoverlapping? In particular, when is $-1<\left(\mathscr{Q}^{+}\right)^{\prime}(p)<0$ ?

In order to keep the computation as simple as possible, we make the following simplifying assumption.

H9: $G_{1}\left(v_{1}, w_{1}\right)$ is independent of $v_{1}$ on $H_{1}$.

Remark 5.2. This assumption simply says that the timescale function $\lambda_{w}\left(v_{1}\right)$ is constant and that $w_{\infty}\left(v_{1}\right) \equiv 1$ on $H_{1}($ see (41)). This assumption is reasonable given the sigmoidal nature of $w_{\infty}\left(v_{1}\right)$ and the role of $\lambda_{w}\left(v_{1}\right)$.

Assumptions $\mathrm{H} 2, \mathrm{H} 4, \mathrm{H} 5$, and $\mathrm{H} 9$ allow us to generalize the ideas of Sect. 3.3 and introduce time coordinates on each branch of both cell 1 and cell 2. Using assumptions $\mathrm{H} 2$ and $\mathrm{H} 5$ the differential equation describing the evolution of $s$ on both $L_{1}$ and $\hat{L}_{1}$ is given by

$$
\frac{d s}{d t}=(1-s) / \lambda_{L}
$$

Using $\mathrm{H} 5$ and $\mathrm{H} 9$ the differential equations on $H_{1}$ reduce to

$$
\begin{aligned}
\frac{d s}{d t} & =-s / \lambda_{H} \\
\frac{d w_{1}}{d t} & =\left(1-w_{1}\right) / \lambda_{w} .
\end{aligned}
$$

The jump function $j: \hat{L}_{1} \mapsto H_{1}$ can be represented in several ways depending on the coordinate system used to parameterize each branch. A natural parameterization of both $\hat{L}_{1}$ and $H_{1}$ is found by using $\left(s, w_{1}\right)$ coordinates. In this coordinate system, which we will refer to as a spatial coordinate system, the jump map, denoted $j_{s}$ in these coordinates, is simply the identity function since a jump from $\hat{L}_{1}$ to $H_{1}$ leaves $\left(s, w_{1}\right)$ unchanged.

Assumptions $\mathrm{H} 2, \mathrm{H} 5$, and $\mathrm{H} 9$ allow a second coordinate system in which $s$ is replaced by a function of $t$. This is a time-based coordinate system. Consider the solution $s_{L}(t ; 0)$ to $(43)$ satisfying $s(0)=0$. This assigns to each $t \geqq 0$ a unique $s$-value in $[0,1)$. Similarly, the solution $s_{H}(t ; 1)$ to $(35)$ satisfying $s(0)=1$ assigns to each $t \geqq 0$ a unique $s$-value in $(0,1] . s_{L}(t ; 0)$ is monotone increasing and $s_{H}(t ; 1)$ is monotone decreasing. Hence each is invertible with inverses given by

$$
s_{L}^{-1}(s)=\int_{0}^{s} \frac{\lambda_{L}}{1-\sigma} d \sigma
$$


and

$$
s_{H}^{-1}(s)=\int_{1}^{s}-\frac{\lambda_{H}}{\sigma} d \sigma
$$

respectively.

It follows that $\hat{L}_{1}$ can be parameterized by $\left(t, w_{1}\right)$ coordinates simply by replacing $s$ with $s_{L}(t ; 0)$ in the spatial coordinate system. This the the time-based coordinates of $\hat{L}_{1}$. Similarly, the time-based coordinates of $H_{1}$ are found by replacing $s$ with $s_{H}(t ; 1)$ in its spatial coordinate system.

Using the commutative diagram below, we can compute the representation of the jump function in time-based coordinates and will denote it by $j_{t}^{1}$.

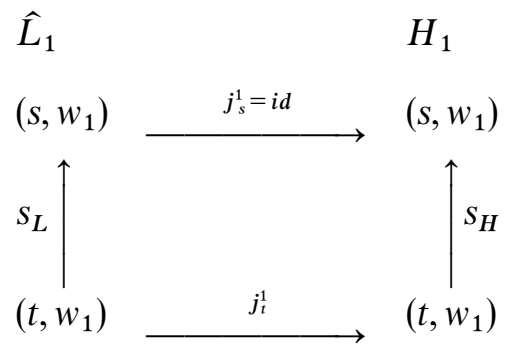

From the commutative diagram in (48) we see that

$$
j_{t}^{1}\left(t, w_{1}\right)=\left(s_{H}^{-1} \circ j_{s}^{1} \circ s_{L}(t ; 0), w_{1}\right)=\left(s_{H}^{-1} \circ s_{L}(t ; 0), w_{1}\right) .
$$

It now follows from (43) and (44) that

$$
C_{\hat{L}_{1} H_{1}}(p):=\frac{d j_{t}^{1}}{d t}=-\frac{\lambda_{H}\left(1-s_{L}(t ; 0)\right)}{\lambda_{L} S_{L}(t ; 0)} \leqq 0
$$

where $p$ is the point on $\hat{L}_{1}$ with time-based coordinates $\left(t, w_{1}\right)$. Thus, as in Sect. 4, the derivative of a jump is simply the compression ratio associated with that jump.

The analysis presented above also applies to a jump from $H_{1}$ to $\hat{L}_{1}$. In this case if $q_{1} \in H_{1}$ then

$$
C_{H_{1} \hat{L}_{1}}\left(q_{1}\right):=\frac{d j_{t}^{1}}{d t}=-\frac{\lambda_{L} s_{H}(t ; 1)}{\lambda_{H}\left(1-s_{H}(t ; 1)\right)} \leqq 0 .
$$

The time-based coordinate systems defined above extend to curves on $\hat{L}_{1}$ and $H_{1}$ that can be parameterized by $s$. For example, the knee $M_{1}(s)$ of $H_{1}$ can be parameterized by time since

$$
M_{1}(s)=M_{1}\left(s_{H}(t ; 1)\right) .
$$


Note that this curve can be parameterized by $t$ even though $M_{1}$ is not invariant under the flow on $H_{1}$. In what follows we will be using these coordinates to parameterize the slow stable manifold, it's image under the jump map on $H_{1}$, and the image of $M_{1}$ under the jump function to $\hat{L}_{1}$.

Using the same ideas as above for cell 2, we introduce time coordinates on $\hat{L}_{2}$ and $\mathrm{H}_{2}$ using the differential equations on each of these branches. On $\hat{L}_{2}$ we use the $d w_{2} / d t$ equation of (41) on $\hat{L}_{2}$ with initial condition $j_{s}^{2}\left(M_{2}\right)$ to define the function $w_{L 2}\left(t ; j_{s}^{2}\left(M_{2}\right)\right)$. This function assigns to each $t \in[0, \infty)$ a $w_{2}$-value and is monotone decreasing.

We take the knee $M_{2}$ of branch $\mathrm{H}_{2}$ as a reference point for introducing time coordinates on $\mathrm{H}_{2}$. However, instead of using the differential equation describing motion on $\mathrm{H}_{2}$ to define time coordinates, we change the sign on the right hand side to give

$$
\frac{d w_{2}}{d t}=-G_{2}\left(v_{2}, w_{2}\right)
$$

This defines time coordinates so that each point is assigned a nonnegative $t$ value. Let $w_{H_{2}}\left(t ; M_{2}\right)$ denote the solution to this equation with initial condition $M_{2}$. If $t$ is the time coordinate of a point $q$ on $\mathrm{H}_{2}$, then $t$ is the time to flow from $q$ to $M_{2} . w_{H_{2}}\left(t ; M_{2}\right)$ is monotone decreasing.

The computation of the time-based coordinate representation of $j^{2}: \hat{L}_{2} \mapsto H_{2}$ is similar to that described above. In particular,

$$
j_{t}^{2}(t)=w_{H 2}^{-1}\left(w_{L 2}\left(t ; j_{s}^{2}\left(M_{2}\right)\right)\right) \text {; }
$$

if $q_{2}$ is a point on $\hat{L}_{2}$ with time coordinate $t_{2}$ and spatial coordinate $w_{2}=w_{L 2}\left(t_{2} ; j_{s}^{2}\left(M_{2}\right)\right)$ then

$$
C_{\hat{L}_{2} H_{2}}\left(q_{2}\right):=\frac{d j_{t}^{2}}{d t}\left(t_{2}\right)=-\frac{\hat{G}_{2}\left(w_{2}\right)}{G_{2}\left(w_{2}\right)} \geqq 0 .
$$

Here $\hat{G}_{2}\left(w_{2}\right)$ is the vector field on $\hat{L}_{2}$ and $G_{2}\left(w_{2}\right)$ is the vector field on $\mathrm{H}_{2}$.

Using the framework established above, we can express the reduced Poincare map 2 in time-based coordinates. Recall that $\mathscr{Q}^{-}=f_{2} \circ f_{1}$ where $f_{1}: S S M \times M_{2} \mapsto L_{1} \times m_{2}$ and $f_{2}: L_{1} \times m_{2} \mapsto S S M \times M_{2}$. Using time-based coordinates the answer to question 1 above is quite simple.

Proposition 5.1. If $p$ is a point on $S S M \times M_{2}$ with time-based coordinate $t$ then $\left(\mathscr{Q}^{-}\right)^{\prime}(t) \equiv 1$.

Proof. Since $p$ is expressed in time coordinates and the $s$ differential equation is the same on $L_{1}$ and $\hat{L}_{1}$ we get

$$
\mathscr{Q}^{-}(t)=t+P_{H}+P_{L}
$$


where $P_{H}$ and $P_{L}$ are the durations of burst and interburst intervals of cell 2 respectively. Since $2^{-}$corresponds to a cycle in which cell 2 does not fire, $P_{H}+P_{L}$ is constant and hence $\left(\mathscr{Q}^{-}\right)^{\prime}(t) \equiv 1$.

Remark 5.3. The fact that $\left(\mathscr{Q}^{-}\right)^{\prime}(t) \equiv 1$ can be interpreted as saying that there is no compression associated with the jumps that comprise $\mathscr{2}^{-}$. This is different than in Sect. 4. This difference follows from the fact that the rate of increase of $s$ on both $L_{1}$ and $\hat{L}_{1}$ is assumed to be identical and the fact that time distance on both $L_{1}$ and $\hat{L}_{1}$ is computed in terms of $s$. Thus each jump between $L_{1}$ and $\hat{L}_{1}$ leaves the time distance between two points unchanged.

By contrast, in Sect. 4 the $w_{1}$-variable was used to define time distance and the $w_{1}$ vector fields differed on $L_{1}$ and $\hat{L}_{1}$ (as they do in the five-dimensional model). Thus each jump associated with $\mathscr{P}^{-}$induced a compression that caused the derivative to be different from 1.

Each of the remaining questions concerns the nature of $\mathscr{2}^{+}$; we begin by describing the time coordinate representation of this function. Recall that $2^{+}=g_{2} \circ g_{1}$ where $g_{1}: S S M \times M_{2} \mapsto M_{1} \times \hat{L}_{2}$ and $g_{2}: M_{1} \times \hat{L}_{2} \mapsto S S M \times M_{2}$. Let $\Omega(s)$ be the parameterization of $S S M$ by $s$. We can express both $g_{1}$ and $g_{2}$ in time coordinates since each of $\Omega(s)$ on $\hat{L}_{1}, M_{1}(s)$ on $H_{1}, j(\Omega(s))$ on $H_{1}$, and $\hat{L}_{2}$ can each be parameterized using time coordinates. Let $p \in S S M$ have time coordinates $\left(t, \Omega\left(s_{H}(t ; 1)\right)\right)$ and let $\tau_{1}(t)$ denote the time to flow from the point $j(p)$ on $H_{1}$ to the knee $M_{1}$. We can express $g_{1}$ in time coordinates by

$$
g_{1}(t)=\left(j_{t}^{1}(t)-\tau_{1}(t), \tau_{1}(t)\right)
$$

where $j_{t}^{1}(t)$ denotes the jump function on cell 1 . The interpretation of (57) is as follows. The first component of $g_{1}$ maps the time coordinate on $\Omega(s)$ to the time coordinate on $M_{1}$. This is found by subtracting $\tau_{1}(t)$, the time to flow to $M_{1}$ from $j(p)$, from the time coordinate of $j(p)$ given by $j_{t}^{1}(t)$. The second component of $g_{1}$ returns the time coordinate on $\hat{L}_{2}$ at the end of the flow portion of $g_{1}$. Since the jump portion of $g_{1}$ maps $M_{2}$ to the reference point $j\left(M_{2}\right)$ on $\hat{L}_{2}$, this is simply $\tau_{1}(t)$.

Now let $\left(q_{1}, q_{2}\right)$ be a point on $M_{1} \times \hat{L}_{2}$ having time coordinates $\left(t_{1}, t_{2}\right)$. Then

$$
g_{2}\left(t_{1}, t_{2}\right)=j_{t}^{1}\left(t_{1}\right)+j_{t}^{2}\left(t_{2}\right)
$$

The first term of this sum converts from time coordinates on $H_{1}$ to time coordinates on $\hat{L}_{1}$ (the jump from $H_{1}$ to $\hat{L}_{1}$ ) and the second term converts from time coordinates on $\hat{L}_{2}$ to $H_{2}$ (the jump from $\hat{L}_{2}$ to $H_{2}$ ). These are added since $j_{t}^{2}\left(t_{2}\right)$ is the time to flow to $M_{2}$ from the point $q_{2}$.

The following proposition addresses question 2 and is the analogue of Proposition 4.3 which gave sufficient conditions for the 
four-dimensional model of Sect. 4 to be in case II. The condition imposed below is similar to that imposed in Proposition 4.3; we demand that the additional time that cell 2 flows on $\mathrm{H}_{2}$ after being released from inhibition be small relative to the standard interburst interval of cell 2. Let $t^{*}$ be the time coordinate of $s^{*}$ on $\hat{L}_{1}$. Then the minimal burst duration of cell 2 is $j_{t}^{2}\left(\tau_{1}\left(t^{*}\right)\right)$.

Proposition 5.2. If $j_{t}^{2}\left(\tau_{1}\left(t^{*}\right)\right)-P_{H}<P_{L}$ then $\mathscr{Q}^{+}\left(t^{*}\right)<\mathscr{2}^{-}\left(t^{*}\right)$.

Proof. Let $g_{1}\left(t^{*}\right)=\left(t_{1}^{*}, t_{2}^{*}\right)$ so that $\mathscr{Q}^{+}\left(t^{*}\right)=j_{t}^{1}\left(t_{1}^{*}\right)+j_{t}^{2}\left(t_{2}^{*}\right)$. Equation (45) implies that $j_{t}^{1}\left(t_{1}^{*}\right) \leqq t^{*}$ (since the flow on $H_{1}$ decreases $s$ ) and thus

$$
\mathscr{2}^{+}\left(t^{*}\right) \leqq t^{*}+j_{t}^{2}\left(t_{2}^{*}\right) \text {. }
$$

Equation (57) implies that $t_{2}^{*}=\tau_{1}\left(t^{*}\right)$ and hence by assumption $j_{t}^{2}\left(\tau_{1}\left(t^{*}\right)\right)<P_{H}+P_{L}$. Therefore

$$
\mathscr{2}^{+}\left(t^{*}\right)<t^{*}+P_{H}+P_{L}=\mathscr{Q}^{-}\left(t^{*}\right) .
$$

We next proceed with question 3 which describes the nature of $\tau_{1}$.

Proposition 5.3. If $\lambda_{H}$ is sufficiently small relative to $\lambda_{w}$, then $\tau_{1}^{\prime}(t)<0$. If $\lambda_{w}$ is sufficiently small relative to $\lambda_{H}$, then $\tau_{1}^{\prime}(t)>0$.

Proof. Let the time coordinate representation of the slow stable manifold be given by $w_{1}=\Omega(s)=\Omega\left(s_{L}(t ; 0)\right)$ and let $p$ have time coordinates $\left(t, \Omega\left(s_{L}(t ; 0)\right)\right.$ on the slow stable manifold. Since $\tau_{1}(t)$ is the time to flow from $j^{1}(p)$ to $M_{1}$ on $H_{1}$ it is implicitly defined by

$$
w_{H}\left(\tau_{1}(t) ; \Omega\left(s_{L}(t ; 0)\right)\right)=M_{1}\left(s_{H}\left(\tau_{1}(t) ; s_{L}(t ; 0)\right)\right)
$$

where $w_{H}\left(t ; w_{0}\right)$ is the solution of (45) with initial condition $w_{0}$. Since the jump from $\hat{L}_{1}$ to $H_{1}$ leaves $s$-coordinates unchanged, $s_{L}(t ; 0)$ is the $s$-coordinate of $j^{1}(p)$ on $H_{1}$ and $j^{1}(p)$ has time coordinate $j_{t}^{1}(t)$ (see (48)). Thus we can use the group property of flows to rewrite (61) as

$$
w_{H}\left(\tau_{1}(t) ; \Omega\left(s_{L}(t ; 0)\right)\right)=M_{1}\left(s_{H}\left(j_{t}^{1}(t)+\tau_{1}(t) ; 1\right)\right) .
$$

Differentiating (62) implicitly yields

$$
\tau_{1}^{\prime}(t)=\frac{\frac{d M_{1}}{d s} \frac{\partial s_{H}}{\partial t} \frac{d j_{t}^{1}}{d t}-\frac{\partial w_{H}}{\partial w_{1}} \frac{d \Omega}{d s} \frac{\partial s_{L}}{\partial t}}{\frac{\partial w_{H}}{\partial t}-\frac{d M_{1}}{d s} \frac{\partial s_{H}}{\partial t}} .
$$

Since $\partial w_{H} / \partial t>0, \partial s_{H} / \partial t<0$ and $M_{1}^{\prime}(s)>0$, the denominator of $(63)$ is positive. Thus the sign of $\tau_{1}^{\prime}(t)$ is determined by the numerator of (63). 
The terms in the numerator of (63) satisfy the following:

1. $d M_{1} / d s>0$ by $(\mathrm{H} 3)$,

2. $\partial s_{H} / \partial t<0$ by $(\mathrm{H} 2)$,

3. $d j_{t}^{1} / d t<0$ by $(50)$,

4. $\partial w_{H} / \partial w_{1}>0$ by (45),

5. $d \Omega / d s>0$ by $(\mathrm{H} 3)$, and

6. $\partial s_{L} / \partial t>0$ by $(\mathrm{H} 2)$.

Only items $2-4$ in the above list depend on $\lambda_{H}$ and $\lambda_{w}$. From (44) we compute that $s_{H}(t ; 1)=\mathrm{e}^{-t / \lambda_{H}}$ and hence

$$
\left.\frac{\partial s_{H}}{\partial t}\right|_{\left(j_{t}^{1}(t)+\tau_{1}(t) ; 1\right)}=\frac{1}{\lambda_{H}} \mathrm{e}^{-\left(j_{t}^{1}(t)+\tau_{1}(t)\right) / \lambda_{H}} .
$$

From (45) we get that $w_{H}\left(t ; w_{1}\right)=1-\left(1-w_{1}\right) \mathrm{e}^{-t / \lambda}$ w and thus

$$
\left.\frac{\partial w_{H}}{\partial w_{1}}\right|_{\left(\tau_{1}(t) ; \Omega\left(s_{L}(t ; 0)\right)\right)}=\mathrm{e}^{-\tau_{1}(t) / \lambda_{w}} .
$$

By substituting these and the expression for $d j_{t}^{1} / d t$ given in (50) we see that the numerator of (63) can be written as

$$
\frac{d M_{1}}{d s} \frac{1-s_{L}(t ; 0)}{\lambda_{L} S_{L}(t ; 0)} \mathrm{e}^{-\left(j_{t}^{1}(t)+\tau_{1}(t)\right) / \lambda_{H}}-\frac{d \Omega}{d s} \frac{\partial s_{L}}{\partial t} \mathrm{e}^{-\tau_{1}(t) / \lambda_{w}} .
$$

We conclude that if $\lambda_{H}$ is sufficiently small relative to $\lambda_{w}$, then $\tau_{1}^{\prime}(t)<0$ and if $\lambda_{H}$ is sufficiently large relative to $\lambda_{w}$, then $\tau_{1}^{\prime}(t)>0$.

We now proceed with question 4 . Since the derivative of each jump is the compression ratio associated with that jump (see (50), (51), and (55)), we get that

and

$$
D g_{1}(t)=\left[C_{\hat{L}_{1} H_{1}}(p)-\tau_{1}^{\prime}(t), \tau_{1}^{\prime}(t)\right]
$$

$$
D g_{2}\left(t_{1}, t_{2}\right)=\left[\begin{array}{l}
C_{\mathrm{H}_{1} \hat{L}_{1}}\left(q_{1}\right) \\
C_{\hat{\mathrm{L}}_{2} \mathrm{H}_{2}}\left(q_{2}\right)
\end{array}\right] .
$$

If $g_{1}(p)=\left(q_{1}, q_{2}\right)$ and $\left(q_{1}, q_{2}\right)$ has time coordinates $\left(t_{1}, t_{2}\right)$ then

$$
\left(\mathscr{Q}^{+}\right)^{\prime}(t)=C_{\hat{L}_{1} H_{1}}(p) C_{H_{1} \hat{L}_{1}}\left(q_{1}\right)-\tau_{1}^{\prime}(t)\left[C_{H_{1} \hat{L}_{1}}\left(q_{1}\right)-C_{\hat{L}_{2} H_{2}}\left(q_{2}\right)\right] .
$$

The first term of this difference is non-negative (see (50) and (51)) and the bracketed quantity of the second term is non-positive (see (51) and (52)). Thus a necessary condition to insure that $\left(\mathscr{Q}^{+}\right)^{\prime}(t) \leqq 0$ is $\tau_{1}^{\prime}(t) \leqq 0$. To insure that $\left(\mathscr{Q}^{+}\right)^{\prime}(t)$ is strictly negative we need to be more careful since $\tau_{1}^{\prime}(t)$ and the compression ratios for cell 1 depend of the rate constant $\lambda_{H}$. 
Proposition 5.4. If $\lambda_{H}$ is sufficiently small then $\left(\mathscr{Q}^{+}\right)^{\prime}(t)<0$.

Proof. Using the definitions of $C_{\hat{L}_{1} H_{1}}$ and $C_{H_{1} \hat{L}_{1}}$ given in (50) and (51) and the definitions of $s_{L}$ and $s_{H}$ derived from (43) and (44) we get that

$$
\lim _{\lambda_{H} \rightarrow 0} C_{\hat{L}_{1} H_{1}}(p) C_{H_{1} \hat{L}_{1}}\left(q_{1}\right)=\lim _{\lambda_{H} \rightarrow 0} \frac{\mathrm{e}^{-t / \lambda_{L}}}{1-\mathrm{e}^{-t / \lambda_{L}}} \frac{\mathrm{e}^{-t_{1} / \lambda_{H}}}{1-\mathrm{e}^{-t_{1} / \lambda_{H}}}=0
$$

and

$$
\lim _{\lambda_{H} \rightarrow 0} C_{H_{1} \hat{L}_{1}}\left(q_{1}\right)=-\lim _{\lambda_{H} \rightarrow 0} \frac{\lambda_{L} \mathrm{e}^{-t_{1} / \lambda_{H}}}{\lambda_{H}\left(1-\mathrm{e}^{-t_{1} / \lambda_{H}}\right)}=0
$$

Thus

$$
\lim _{\lambda_{H} \rightarrow 0}\left(Q^{+}\right)^{\prime}(t)=C_{\hat{L}_{2} \mathrm{H}_{2}}\left(q_{2}\right) \lim _{\lambda_{H} \rightarrow 0} \tau_{1}^{\prime}(t) .
$$

From (55) $C_{\hat{\mathrm{L}}_{2} \mathrm{H}_{2}}\left(q_{2}\right)>0$ and by Proposition 5.3

$$
\lim _{\lambda_{H} \rightarrow 0} \tau_{1}^{\prime}(t) \leqq 0 .
$$

We are done if we can show that this limit is strictly negative. Only $\partial s_{H} / \partial t$ and $d j_{t}^{1} / d t$ depend on $\lambda_{H}$ in (63). Using (43), (44), and (50) we get

$$
\left.\lim _{\lambda_{H} \rightarrow 0} \frac{\partial s_{H}}{\partial t}\right|_{j_{t}^{1}(t)+\tau_{1}(t)}=\lim _{\lambda_{H} \rightarrow 0} \frac{\mathrm{e}^{-\left(j_{t}^{1}(t)+\tau_{1}(t)\right) / \lambda_{H}}}{\lambda_{H}}=0
$$

and

$$
\lim _{\lambda_{H} \rightarrow 0}\left(\left.\frac{\partial s_{H}}{\partial t}\right|_{j_{t}^{1}(t)+\tau_{1}(t)}\right) \frac{d j_{t}^{1}}{d t}=\lim _{\lambda_{H} \rightarrow 0} \frac{1-s_{L}(t ; 0)}{\lambda_{L} s_{L}(t ; 0)} \mathrm{e}^{-\left(j_{t}^{1}(t)+\tau_{1}(t)\right) / \lambda_{H}}=0 .
$$

Thus

$$
\lim _{\lambda_{H} \rightarrow 0} \tau_{1}^{\prime}(t)=-\frac{\frac{\partial w_{H}}{\partial w_{1}} \frac{d \Omega}{d s} \frac{\partial s_{L}}{\partial t}}{\frac{\partial w_{H}}{\partial t}}<0
$$

and $\left(\mathscr{Q}^{+}\right)^{\prime}(t)<0$ if $\lambda_{H}$ is sufficiently small.

In the special case of instantaneous reset, (69) reduces to a simpler form. In this scenario, the $s$ coordinate of $g_{1}$ is identically 0 at the conclusion of the flow portion of $g_{1}$. This corresponds to $s_{H}(t ; 1)=0$ in (51) so that

$$
C_{H_{1} \hat{L}_{1}}\left(t_{1}\right)=0 .
$$


Substituting this into (69) yields

$$
\left(\mathscr{Q}^{+}\right)^{\prime}(t)=\tau_{1}^{\prime}(t) C_{\hat{L}_{2} H_{2}}\left(t_{2}\right) .
$$

Since instantaneous reset corresponds to $\lambda_{H} \rightarrow 0$ it follows from Proposition 5.3 that $\left(\mathscr{Q}^{+}\right)^{\prime}(t)<0$.

We conclude with the answer to question 5 .

Proposition 5.5. If $\lambda_{H}$ is sufficiently small and $\lambda_{L}$ is sufficiently large then $-1<\left(\mathscr{Q}^{+}\right)^{\prime}(t)<0$ and thus 2 is nonoverlapping.

Proof. Equations (72) and (76) imply that

$$
\lim _{\lambda_{H} \rightarrow 0}\left(Q^{+}\right)^{\prime}(t)=-\frac{\frac{\partial w_{H}}{\partial w_{1}} \frac{d \Omega}{d s} \frac{\partial s_{L}}{\partial t}}{\frac{\partial w_{H}}{\partial t}} C_{\hat{L}_{2} H_{2}}\left(q_{2}\right) .
$$

Using the solutions to (34) and (36) we get

$$
\lim _{\lambda_{H} \rightarrow 0}\left(\mathscr{Q}^{+}\right)^{\prime}(t)=\frac{\left(1-\Omega\left(1-\mathrm{e}^{-t / \lambda_{L}}\right)\right) \Omega^{\prime}\left(1-\mathrm{e}^{-t / \lambda_{L}}\right) \mathrm{e}^{-t / \lambda_{L}}}{\lambda_{w} \lambda_{L}} C_{\hat{L}_{2} H_{2}}\left(q_{2}\right) .
$$

Hypothesis $(\mathrm{H} 3)$ guarantees that $\Omega^{\prime}(0) \neq 0$ and thus by choosing $\lambda_{L}$ sufficiently large we can make this quantity less than 1 in absolute value. Thus for $\lambda_{H}$ sufficiently small and $\lambda_{L}$ sufficiently large $-1<\left(\mathscr{Q}^{+}\right)^{\prime}(t)<0$.

\section{Discussion}

The calculations used in this paper use a "singular Poincare map," which combines estimates from the flow on a slow manifold with estimates of "compression" across a jump between branches of a slow manifold. The notion of "compression across a jump" was central in the work of Somers and Kopell [31] with two-dimensional relaxation oscillators; in that case, however, the flow on each slow branch can be seen as a pair of uncoupled one-dimensional flows. In the current problem and in related work [32], there is more than one slow variable in one or more of the cells and the emergent dynamics depends critically on the interactions of the slow variables. Other related papers that produce dynamics similar to the ones in this paper from voltagegated conductance equations include $[27,34,36]$. These are all simulation papers.

In this paper, the crucial slow variables are the recovery time of each of the cells, and the rise time and reset time of the slow ionic 
current $i_{h}$. All of these processes are slow relative to the rate of change of the voltage, but there are not orders of magnitude difference among the slow rates themselves. However, more subtle differences in these rates are important in determining the dynamics. A sufficiently slow rise time of the $i_{h}$ (relative to the recovery time of cell 1) leads to a singular Poincare map that is essentially one-dimensional, while a sufficiently fast reset time of that current, during the activation of cell 1, leads to the timing regulation discussed in Sect. 5 and below.

Computations similar in spirit were used by Terman et al. [32] in their work on the effects of slowly decaying inhibition. Again, there are several slow variables whose rates of change have similar orders of magnitude, and whose sizes are critical in determining some property of the system (in that case, whether the synchronous solution is stable). In that paper, the relevant slow processes are the decay time of the slow inhibition, recovery time of the cells, and the burst time of the cells; in different parameter regimes, different combinations of those parameters govern stability.

The reduction and analysis allow one to see with much greater clarity how the time scales interact to produce behavior with potentially important functional consequences. In the current paper, the functional issue concerns the constancy of the burst time of the slow cell (cell 1) from cycle to cycle. Though the data that inspired this work were produced in circumstances that do not necessarily occur in physiological ranges, they raise issues related to the coordination of the fast pyloric rhythm and the slow gastric rhythm in the stomatogastric ganglion [35]. Recent models [27] suggest that the slow rhythm depends for its existence on the coupling with the faster rhythm; to the extent that the burst length of the gastric rhythm helps time the neurons of the circuit, the timing regulation is an important issue. Furthermore, burst length can also affect the size of interburst intervals, due to currents that prolong the latter when the former is long [36]; thus regulation of burst lengths can be important in other aspects of timing regulation as well.

Figure 28 illustrates the change in burst length when coordination is not $n: 1$. The burst length of cell 1 (part $\mathrm{A}$ ) is long when it fires after 2 bursts of cell 2, but is shorter when it fires after only one burst of cell 1. Figure 28 was generated using a modification of the equations given in the Appendix in the absence of $i_{h}$. For cell 1 the function $\lambda_{w}\left(v_{1}\right)$ was modified in such a way as to increase the burst length while leaving the interburst time relatively unchanged. This increases the time between points on branch $H_{1}$ and thus reduces the compression associated with the jump from branch $\hat{L}_{1}$ to $H_{1}$. The affect of this is two-fold. First it increases the parameter range in which non-integer 

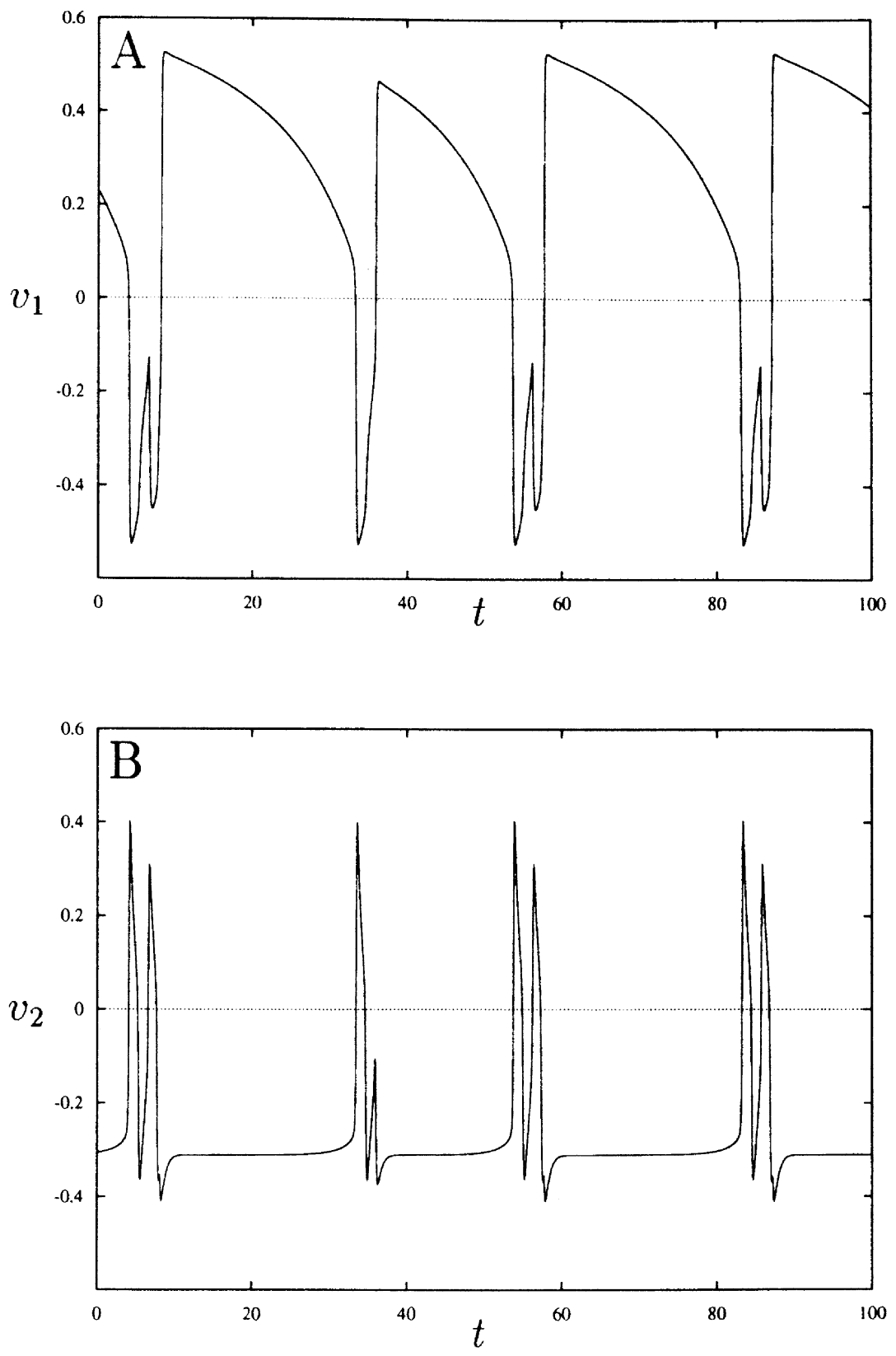

Fig. 28. A change in burst duration. A: The voltage of cell $1\left(v_{1}\right)$ plotted as a function of time $(t)$. B: The voltage of cell $2\left(v_{2}\right)$ plotted as a function of time $(t)$. In cases of $n$ : $m$ coordination the burst length of the excitable cell (cell 1) can change from burst to burst. Note that when cell 1 fires after 2 bursts of cell 2 the burst length is long, but when cell 1 fires after 1 burst of cell 2 the burst length is short. 
subharmonics can be found. Secondly, it makes the change in burst length from burst to burst more significant.

The reduction and analysis also allow one a clearer picture of how changes in biophysical parameters results in changes in the dynamics. In addition to insights about $n: 1$ versus $n: m$ coordination, the reduced map, plus the reduction procedure, allows one to see what determines the $n$ in the $n: 1$ regime. In [23] it is shown that a one parameter family of non-overlapping maps having the qualitative features of the map $\mathscr{2}$ (see Fig. 26B) undergoes a sequence of period-adding bifurcations as the discontinuity of the map is shifted rightward. In addition, it is shown that intervals of bistability occur prior to each bifurcation.

The discontinuity in the reduced Poincare map 2 described in this paper occurs at the point $s^{*}$ which is the intersection of the slow stable manifold on $\hat{L}_{1}$ and the projection onto $\hat{L}_{1}$ of the knee $m_{1}(s)$ on branch $L_{1}$. Thus, in the parameter regime where $-1<\left(\mathscr{Q}^{+}\right)^{\prime}(t)<0$, periodadding bifurcations occur as the value of $s^{*}$ is increased. In [22] it is shown that $s^{*}$ increases as one increases the parameter $I$ representing the level of hyperpolarizing current applied to cell 1 (in the limiting case where $S S M=\hat{E}_{1}(s)$ ). Period-adding and hysteresis was observed in the numerical investigation of the model equations described in the Appendix $[21,22]$ when the parameter $I$ was increased.

\section{Appendix}

The equations used in the simulations are slight modifications of the non-dimensional Morris-Lecar equations [26, 29]. We denote the voltages of the LP and PD cells by $v_{1}$ and $v_{2}$ respectively. The $w_{1}$ and $w_{2}$ variables are loosely modeled on the activation of an outward current. The level of $i_{h}$ is called $s$. The equations used in these simulations are slight modifications of those used in [21] and are

$$
\begin{aligned}
d v_{1} / d \tau & =F_{1}\left(v_{1}, w_{1} ; I_{1}\right)+H\left(v_{1}, s\right)+\alpha m_{\infty}\left(v_{2}\right)\left(v_{s y n}-v_{1}\right) \\
d w_{1} / d \tau & =\varepsilon G_{1}\left(v_{1}, w_{1}\right) \\
d s / d \tau & =\varepsilon\left[s_{\infty}\left(v_{1}\right)-s\right] / \lambda_{s}\left(v_{1}\right) \\
d v_{2} / d \tau & =F_{2}\left(v_{2}, w_{2} ; I_{2}\right)+\alpha m_{\infty}\left(v_{1}\right)\left(v_{s y n}-v_{2}\right) \\
d w_{2} / d \tau & =\varepsilon G_{2}\left(v_{2}, w_{2}\right) .
\end{aligned}
$$

The basic currents and recovery dynamics have the form:

$$
\begin{aligned}
F_{i}(v, w ; I) & =I+g_{k} w\left(v_{k}-v\right)+g_{\ell}\left(v_{\ell}-v\right)+g_{c a} m_{\infty}(v)(1-v), \\
G_{i}(v, w) & =\left[w_{\infty}\left(v ; \bar{v}_{3}\right)-w\right] / \lambda_{w}(v)
\end{aligned}
$$


for $i=1,2$. The functions $m_{\infty}(v), w_{\infty}\left(v ; \bar{v}_{3}\right)$, and $\lambda_{w}(v)$ are given by

$$
\begin{aligned}
m_{\infty}(v) & \equiv \frac{1}{2}\left(1+\tanh \left(\frac{v-\bar{v}_{1}}{\bar{v}_{2}}\right)\right), \\
w_{\infty}\left(v ; \bar{v}_{3}\right) & \equiv \frac{1}{2}\left(1+\tanh \left(\frac{v-\bar{v}_{3}}{\bar{v}_{4}}\right)\right), \\
\lambda_{w}(v) & \equiv 1 / \cosh \left(\frac{v-\bar{v}_{5}}{2 \bar{v}_{4}}\right) .
\end{aligned}
$$

The values of the cell independent constants are $\bar{v}_{1}=-0.01, \bar{v}_{2}=0.15$, $\bar{v}_{4}=0.3, \bar{v}_{5}=0.22, g_{k}=2, v_{k}=-0.7, g_{\ell}=0.5, v_{\ell}=-0.5, g_{c a}=10.1$, $\varepsilon=0.1, \alpha=0.5$ and $v_{\text {syn }}=-0.1$.

Different values of $\bar{v}_{3}$ were used for cell 1 and cell 2 to insure that cell 2 was not able to burst when inhibited by cell 1 (see H1). For cell 1 we let $\bar{v}_{3}=0.12$ and for cell 2 we let $\bar{v}_{3}=0.0$. We set $I_{2}=0.35$ to insure that cell 2 oscillates in the absence of inhibition; $I_{1}$ was varied.

The effect of $i_{h}$ has the form

$$
H\left(v_{1}, s\right)=g_{s} s\left(v_{s}-v_{1}\right)
$$

with $g_{s}=0.5$ and $v_{s}=0.3$. The dynamics of the $i_{h}$ current are defined by the functions $\lambda_{s}(v)$ and $s_{\infty}(v)$ which are given by

$$
\begin{aligned}
\lambda_{s}(v) & =\frac{33.33}{\left(1+\mathrm{e}^{\left(v-v_{7}\right) / v_{6}}\right)}, \\
s_{\infty}(v) & =\frac{1}{1+\mathrm{e}^{\left(v-v_{9}\right) / v_{s}}}
\end{aligned}
$$

where $\bar{v}_{6}=0.04, \bar{v}_{7}=0.05, \bar{v}_{8}=0.075$, and $\bar{v}_{9}$ was varied.

The numerical simulations were done using the software PHASEPLANE [7] and dstool [12]. The integration method employed was either a fourth order Runge-Kutta with small step size or the variable step size Gear's method.

\section{References}

1. Angstadt, J. D. and Calabrese, R. L. (1989) A hyperpolarization-activated inward current in heart interneurons of the medicinal leech. J. Neurosci., 9: 2846-2857

2. Arnold, V. I. (1989) Mathematical Methods of Classical Mechanics. Graduate Texts in Mathematics, 60, Springer-Verlag, Berlin

3. Bonet, C. (1987) Singular perturbation of relaxed periodic orbits. J. Diff. Eqns., 66: 301-339 
4. Chialvo, D. R., Michaels, D. and Jalife, J. (1990) Supernormal excitability as a mechanism of chaotic dynamics of activation in cardiac Purkinje fibers. Circ. Res., 66: 525-545

5. Devaney, R. (1989) An Introduction to Chaotic Dynamical Systems, 2nd ed., Addison-Wesley, Redwood City

6. Ermentrout, G. B. (1981) $n: m$ Phase-locking of weakly coupled oscillators, J. Math. Biol., 12: 327-342

7. Ermentrout, G. B. (1989) PHASEPLANE, Version 3.0. Brooks/Cole Publishing

8. Friesen, W. (1994) Reciprocal inhibition, a mechanism underlying oscillatory animal movements, Neurosci. Behavior, 18: 547-553

9. Glass, L. and Mackey, M. (1979) A simple model for phase locking of biological oscillators, J. Math. Biol., 7: 339-352

10. Golowasch, J., Buchholtz, F., Epstein, I.R. and Marder, E. (1992) The contribution of individual ionic currents to the activity of a model stomatogastric ganglion neuron. J. Neurophysiol., 67: 341-349

11. Golowasch, J. and Marder, E. (1992) Ionic currents of the lateral pyloric neuron of the stomatogastric ganglion of the crab, J. Neurophysiol., 67: 318-331

12. Guckenheimer, J., Myers, M. R., Wicklin, F. J., and Worfolk, P. A. (1991) dstool: A Dynamical System Toolkit with an Interactive Graphical Interface. Center for Applied Mathematics, Cornell University

13. Harris-Warrick, R. M. and Marder, E. (1991) Modulation of neural networks for behavior, Ann. Rev. Neurosci., 14: 39-57

14. Harris-Warrick, R., Marder, E., Selverston, A. and Moulins, M. eds (1992) Dynamic biological networks: the stomatogastric nervous system, MIT Press

15. Hooper, S. Unpublished data

16. Keener, J. P., (1980) Chaotic behavior in piecewise continuous difference equations, Trans. of the AMS, 261: 589-604

17. Kiehn, O. and Harris-Warrick, R.M. (1992) 5-HT modulation of hyperpolarization-activated inward current and calcium-dependent outward current in crustacean motor neuron. J. Neurophysiol., 68: 496-508

18. Kopell, N. and Somers, D. (1995) Anti-phase solutions in relaxation oscillators coupled through excitatory interactions, J. Math. Biol., 33: 261-280

19. Levi, M. (1981) Qualitative analysis of the periodically forced relaxation oscillations, Mem. AMS, 214: 1-147

20. Levi, M. (1990) A period-adding phenomenon, SIAM J. Appl. Math, 50: 943-955

21. LoFaro, T., Kopell, N. Marder, E. and Hooper, S. (1994) Subharmonic Coordination in Networks of Neurons with Slow Conductances. Neural Computation, 6: 69-84

22. LoFaro, T. (1994) A period adding bifurcation in a pair of coupled neurons, doctoral thesis, Boston University

23. LoFaro, T. (1996) Period adding bifurcations in a one parameter family of interval maps, Mathl. Comput. Modelling, 24: 27-41

24. McCormick, D. A. and Pape, H. C. (1990) Noradrenergic and serotonergic modulation of a hyperpolarization-activated cation current in thalamic relay neurons. J. Physiol., 431: 319-342

25. Mischenko, E. F. and Rosov, N. (1980) Differential Equations with Small Parameters and Relaxation Oscillations, Plenum Press, New York

26. Morris, H. and Lecar, C. (1981) Voltage oscillators in the barnacle giant muscle fiber. Biophysical J., 35: 193-213 
27. Nadim, F. and Manor, Y., Nusbaum, M., and Marder, E. (1998) Frequency regulation of a slow rhythm by fast periodic input in a model gastric mill network, J. Neurosci., 18: 5053-5067

28. Perkel, D. H. and Mulloney, B. (1974) Motor pattern production in reciprocally inhibitory neurons exhibiting postinhibitory rebound. Science, 185: 181-183

29. Rinzel, J. and Ermentrout, G. B. (1989) Analysis of neural excitability and oscillations, in Methods in Neuronal Modeling: From Synapses to Networks, ed. C. Koch and I. Segev. MIT Press, 135-163

30. Skinner, F. K., Kopell, N. and Marder, E. (1994) Mechanisms for oscillation and frequency control in reciprocal inhibitory model neural networks, J. Comp. Neurosci., 1: 69-87

31. Somers, D. and Kopell, N. (1993) Rapid synchronization through fast threshold modulation, Biol. Cyber., 68: 393-407

32. Terman, D., Bose, A., and Kopell, N. (1997) Functional reorganization in thalamocortical networks: Transition between spindling and delta sleep rhythms, Proc. Natl. Acad. Sci. USA, 93: 15417-15422

33. van Vreeswijk, L. Abbott and Ermentrout, G. B. (1994) When inhibition, not excitation synchronizes neural firing, J. Comp. Neurosci, 1: 313-321

34. Wang, X. J. and Rinzel, J. (1992) Alternating and synchronous rhythms in reciprocally inhibitory model neurons, Neural Comp., 4: 84-97

35. Weimann, J. M., Meyrand, P., and Marder, E. (1991) Neurons that form multiple pattern generators: Identification and multiple activity patterns of gastric/pyloric neurons in the crab stomatogastric system. J. Neurophysiol., 65: 111-122

36. Wiemann, J., Skiebe, P., Heinzel, H.-G., Soto, C., Kopell, N., Jorge-Rivera, J. C., and Marden, E. (1996) Modulation of oscillator interactions in the crab stomatogastric ganglion by crustacean cadioactive peptide, J. Neurosci., to appear

37. Xie, M., Othmer, H. G. and Watanabe, M. (1996) Resonance in excitable systems under step-function forcing II. Subharmonic solutions and persistence, Physica D, 98: $75-110$ 